\title{
Squark Flavor Violation at the LHC
}

\author{
Graham D. Kribs ${ }^{a}$, Adam Martin ${ }^{b}$ and Tuhin S. Roy ${ }^{a}$ \\ ${ }^{a}$ Department of Physics and Institute of Theoretical Science, \\ University of Oregon, Eugene, OR 97403 \\ ${ }^{b}$ Department of Physics, Sloane Laboratory, Yale University, New Haven, CT 06520
}

\begin{abstract}
We consider the prospects for measuring squark flavor violation through the signal of single top production at the LHC. We study this signal in the context of $R$-symmetric supersymmetry, which allows for large flavor violation in the squark sector, however the results can also be generalized to the MSSM. The single top signal arises from squark pair production in which one squark decays to a top and gaugino, whereas the other squark decays to a non-top quark and gaugino. We study three decay patterns: (I) squark decay into a quark and neutralino LSP; (II) squark decay into a quark and neutralino NLSP, with subsequent decay of the NLSP to a photon and gravitino; (III) squark decay into a quark and chargino NLSP, with subsequent decay of the NLSP to a $H^{ \pm} / W^{ \pm}$and gravitino. Case II is the most promising, when the NLSP decay is prompt, since every event contains two hard photons that can be used to tag the events, reducing the background to a negligible level. Case I is promising if the neutralino LSP is bino-like. We carefully consider large SM backgrounds and identify a series of cuts to isolate the signal. Case III can occur in the minimal $R$-symmetric supersymmetric standard model (MRSSM) with Higgsino-like lightest gauginos. Due to the large Higgs coupling, squarks preferentially decay to top quarks, substantially reducing the potential flavor violating signal. Nevertheless, the flavor violating signal might still be identifiable if the chargino NLSP is long-lived.
\end{abstract}




\section{Introduction}

The potential misalignment of flavor between supersymmetry breaking parameters and the ordinary CKM matrix leads to the possibility of supersymmetry-induced flavor violation $[1,2]$. In general the size of this flavor violation, within the minimal supersymmetric standard model (MSSM), far exceeds the bounds from the myriad observations and searches for flavor violation in the quark and lepton sector [3]. Constraints on flavor violation are strongest between the first and second generation, though substantial constraints also exist between the third generation and the light generations. The top sector is the least constrained, but also the most interesting for collider physics, since tops can be identified given their kinematics and decay pattern.

Recently, a new approach to weak scale supersymmetry that incorporates an (exact or approximate) $R$-symmetry [4], suggests large flavor violation in the supersymmetry breaking parameters may be present without exceeding the flavor-violating bounds [4,5]. This is possible for several reasons: left-right squark and slepton mixing is absent; the gaugino masses $M$ can be naturally $4 \pi / g$ heavier than the scalar masses; and several flavor-violating operators are more suppressed than in the MSSM due to the absence of $R$-violating operators. In this paper, we study one particularly interesting collider signal of flavor violation within the context of an $R$-symmetric model: single top production from squark pair production in which one squark decays to a top and gaugino, while the other squark decays to a non-top quark and gaugino. Interestingly, another source of single top can result from sgluons in $R$-symmetric models, as was recently shown by [6].

Single top is an important process in the Standard Model (SM) [7]. Beyond the Standard Model, in the context of supersymmetry, several groups have explored a single top signal with or without additional flavor violation in the squark mass matrices [8-15] as well as resulting from $R$-parity violation [16-19]. Unlike the approaches in these papers, however, our single top signal does not suffer from substantial restrictions from other flavor-violating processes. The presence of an $R$-symmetry suggests large flavor-violation can be probed in a variety of ways involving sleptons as well as squarks. Nevertheless, we make no attempt at an exhaustive study of flavor violation.

Our focus is on the shortest possible decay chains of squarks: pair-produced squarks that decay into different flavors of quarks and the lightest gaugino. We consider three basic scenarios: (I) the "collider-equivalent" lightest supersymmetric particle (LSP) is a neutralino, (II) the nextto-LSP (NLSP) is a neutralino that decays within the detector to a photon and a gravitino LSP, and (III) the NLSP is a chargino that may or may not decay (within the detector) to a gravitino LSP.

Cases II and III arise when the scale of mediation of supersymmetry breaking is low [20] or as a consequence of a strongly coupled hidden sector [21-23]. In an $R$-symmetric model, $R$-symmetry itself may be broken explicitly in the hidden sector to cancel the cosmological constant. This explicit breaking, communicated via anomaly-mediation to the visible sector, leads to suppressed contributions to $R$-violating supersymmetry breaking parameters. The size of this contribution is proportional to the gravitino mass, which cannot exceed the weak scale 
to ensure the $R$-violation in the visible sector, proportional to $\alpha / 4 \pi \times$ the gravitino mass, is sufficiently small. Given a much smaller mass for the gravitino, where it becomes the LSP, the induced $R$-violation automatically becomes safe. Two interesting scenarios for a signal of flavor violation result within the MRSSM with a gravitino LSP: one with a neutralino next-to-lightest supersymmetric particle (NLSP); the other, a chargino NLSP. Depending on the strength of the interaction between the NLSP and the LSP, the NLSP can be long lived. The characteristic signals depend sensitively on the lifetime of the NLSP.

Finally, our MRSSM analysis can also be applied to the MSSM in a partially $R$-symmetric limit, which may well be of interest in its own right. As a reminder, $R$-symmetry forbids leftright mass mixings among squarks and sleptons, and also implies gauginos are Dirac fermions. (A brief review of the MRSSM and its characteristics is provided in the Appendix of this paper.) At the LHC, squark two-body decay into a Dirac gaugino LSP is indistinguishable from squark decay two-body decay into a Majorana gaugino LSP. Then in the MSSM, left-right mixing is proportional to $m_{f}\left(A_{f}-\mu / \tan \beta\right)$ or $m_{f}\left(A_{f}-\mu \tan \beta\right)$ for up-type or down-type sfermions, and thus can vanish for particular choices of parameters. It is in this limit that our results apply to the MSSM.

The organization of this paper is as follows. In Sec. 2 we outline the three classes of signals that we consider in this paper. We then proceed to analyze each case in subsequent sections. In Sec. 3 we consider the signal top signal with a neutralino LSP. In Sec. 4 we consider the signal top signal with a neutralino NLSP that decays within the detector to a gravitino and photon. In Sec. 5 we consider the signal top signal with a chargino NLSP. Finally, we conclude in Sec. 6 .

\section{Squark Flavor Violation: 3 Cases of 3 Signals}

We are concerned exclusively with squark production and decay at colliders, specifically at the LHC. Consequently, for us "LSP" always refers to "collider-equivalent LSP". That is, the last particle in the supersymmetry decay chain that escapes the detector. This means that we treat a strict neutralino LSP the same as a neutralino collider-equivalent LSP where the latter decays into a gravitino well outside the detector.

There are three separate scenarios with a signal of squark flavor violation that we focus on in this paper:

I: The lightest neutralino is a collider-equivalent LSP. The shortest decay chain possible is for the squark to decay directly into a quark plus neutralino. Within this class of processes, we examine the flavor-violating signal of a single top, i.e., we select events that contain one top quark and one quark of different flavor. Top is identified by the detection of its decay products ( $b$ and a leptonic decay of $W$ ). The specific collider signal is thus one lepton, one tagged $b$ jet, and one other jet. We look for flavor violation in single top for three reasons: first, flavor violation in the up sector, especially flavor violation involving the third generation, is relatively unconstrained. Second, the hard lepton from $W$-decay 
provides a good trigger for these events and an effective way to reduce the immense QCD background. Lastly, $b$-jets can be tagged most efficiently among all jets.

II: The lightest neutralino is the NLSP, which decays within the detector to a gravitino LSP* The NLSP decays into a gravitino and a photon or a $Z$. Decays to a $Z$ are typically kinematically suppressed compared to the decays to a photon, and thus we will focus on signals containing two hard photons plus missing energy. For supersymmetric single-top production, the parton-level final state is now $b j \ell \gamma \gamma+\mathbb{E}_{T}$. However, because the hard photons provide a reliable trigger and excellent background discrimination, we do not require a lepton to suppress SM background in this scenario. Therefore we are free to consider flavor-violating processes other than single-top production: For example, we may also look at flavor violation in the sbottom sector $p p \rightarrow \tilde{q}_{d} \tilde{q}_{d}^{*} \rightarrow b+d+\chi_{1} \bar{\chi}_{1}$, in which case the final state of interest is $b j \gamma \gamma+\mathbb{E}_{T}$.

III: The chargino is the NLSP, and the gravitino is the LSP. The main difference from Case II is that if the chargino is long-lived, it produces tracks inside the detector. Accordingly, three different sub-cases arise depending on the decay length of the chargino:

III.a: The decay of the chargino is prompt. In this case, the single top signal arises from down-squark pair production into one $t$ (decaying into a $b$ and $W$ ) and one jet with two $H^{ \pm} / W^{ \pm}$. Up-squark pair-production gives a similar signal, except that the $b$ is produced directly, and thus there is no additional $W$ from top decay.

III.b: The chargino is long-lived, but decays inside the detector.

III.c: The chargino decays outside the detector, producing tracks of charged heavy particles escaping the detector.

In the next few sections we systematically analyze these cases in the context of observing the single top signal of squark flavor violation.

\section{Case I: A Neutralino LSP}

\subsection{Flavor Violation in Single Top: Setup and Feasibility}

Single top production is widely understood to be an important process as it allows a direct test of the unitarity of the CKM matrix. In the SM, however, single top production proceeds only through electroweak interactions and, depending on the production channel, it is also suppressed by the $b$-quark PDF, CKM angles, or phase space. Thus, this provides an opportunity for a beyond the SM signal to be seen in this channel at the LHC. In our scenario, the BSM-induced single top signal can receive significant enhancement due to the flavor-violating elements in the scalar mass matrices in the MRSSM. Our goal is to isolate this signal from among the SM and detector backgrounds.

*Even if the neutralino decay is not prompt, as long as the decay happens before the electromagnetic calorimeter, the resulting photon may be observed. 
The signal events arise due to the following processes in the MRSSM

$$
p+p \rightarrow\left\{\begin{array}{l}
\tilde{u}_{L_{a}}+\tilde{u}_{L_{a}}^{*} \\
\tilde{u}_{R_{a}}+\tilde{u}_{R_{a}}^{*}
\end{array}\right\} \rightarrow \text { top }+ \text { jet }+\chi_{1}+\bar{\chi}_{1} \rightarrow W+b+\text { jet }+\chi_{1}+\bar{\chi}_{1},
$$

where we used notation from the Appendix: the up-type squarks $\tilde{u}_{L_{a}}$ and $\tilde{u}_{R_{a}}$ are mass eigenstates and $\chi_{1}$ is the lightest neutralino of the MRSSM. For this Case, $\chi_{1}$ escapes the detector, giving rise to additional missing energy. We require that the $W$ decays leptonically for triggering purposes and to suppress multijet QCD backgrounds.

This flavor-violating signal requires that the two squarks decay differently - one decays to top, and the other to an up or charm quark. Both decay modes must have significant branching ratios. A necessary condition for a significant number of signal events is then the presence of large mixing angles in the squark mass matrices. In order to simplify the discussion, we take the squark mixing matrix to be

$$
U_{\tilde{u}_{L}}=\left(\begin{array}{ccc}
\cos \theta_{L} & 0 & \sin \theta_{L} \\
0 & 1 & 0 \\
-\sin \theta_{L} & 0 & \cos \theta_{L}
\end{array}\right), \quad U_{\tilde{u}_{R}}=\left(\begin{array}{ccc}
\cos \theta_{R} & 0 & \sin \theta_{R} \\
0 & 1 & 0 \\
-\sin \theta_{R} & 0 & \cos \theta_{R}
\end{array}\right)
$$

so that we have mixing between two species of the squarks only. Sfermion mixing matrices are defined in App. A.1.2, while the Lagrangian written in the mass eigenstate basis incorporating these mixing matrices can be found in App. A.2. In the following, we will assume the mixing angle to be maximal. The size of our signal, however, can be simply scaled with the mixing angle given otherwise identical kinematics.

The presence of a large mixing angle, by itself, is not sufficient to yield the flavor-violating signal we seek. The squark masses also play a crucial role - they determine whether or not decay channels are kinematically open. Throughout this paper we assume squark masses are large enough such that decays to top and the lightest neutralino is not kinematically forbidden. More importantly, the branching ratios depend crucially on the composition of $\chi_{1}$, i.e., how much bino, neutral wino, and neutral Higgsino is present. There are thus three distinct limits:

a: The lightest neutralino is mostly a bino. Such a scenario arises when $\left.M_{1} \ll \mu_{u}, \mu_{d}, M_{2}\right\rfloor^{\dagger}$ Here $M_{1}, M_{2}, \mu_{u}, \mu_{d}$ are the $R$-symmetric bino, wino, up-type Higgsino and down-type Higgsino masses, defined in App. A.1.1.

b: The lightest neutralino is mostly a neutral Higgsino. This occurs if either or both of $\mu_{u}, \mu_{d}$ are much smaller than $M_{1}$ and $M_{2}$.

c: The lightest neutralino is mostly a wino. This occurs when $M_{2} \ll \mu_{u}, \mu_{d}, M_{1}$. This scenario is disfavored when $M_{2}<1 \mathrm{TeV}$, since a vev develops for the scalar $\mathrm{SU}(2)$ triplet, which leads to an excessive contribution to $\Delta \rho[4]$. Also, a light wino in the presence of flavor violation in the up sector also could lead to sizable contributions to FCNCs such as $K^{0}-\bar{K}^{0}$ mixing. We do not consider this possibility further.

${ }^{\dagger}$ As an aside, this is precisely the mass ordering that was needed in Ref. [25] for a Dirac bino to explain the PAMELA positron ratio excess [26]. 

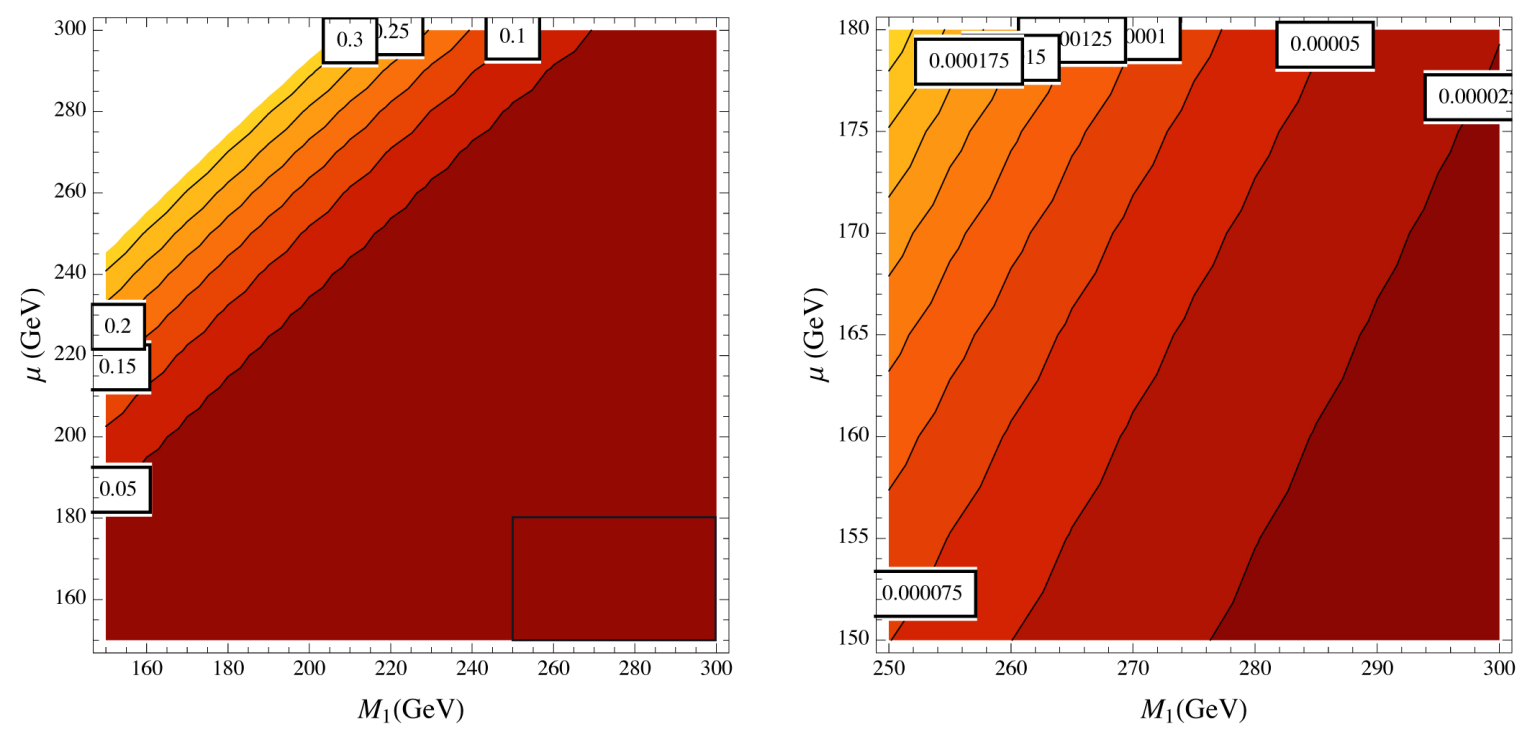

Figure 1: Contours of $r_{\tilde{u}_{L}}$ in the MRSSM for $\tan \beta=10$ and $M_{2}=1 \mathrm{TeV}$. The rightmost figure is a magnification of the rectangular region indicated in the bottom-right corner of the left figure. It shows the rapid decrease of $r$ with increasing $M_{1} / \mu$.

The difference in the first two limits can be easily seen in the gaugino-squark-quark couplings given in the Appendix (c.f., Eq. A-21); in this equation, neutralinos are in electroweak gauge eigenstates). The only quark which couples to up-type squarks in the squark-quark-Higgsino interactions is the top quark. This implies that, if the lightest neutralinos are Higgsinos (in the notation of the Appendix, $\bar{N} P_{3}$ and $\bar{N} P_{4}$ ), the two body decay of up-type squarks to the lightest neutralino always proceed to a top quark - regardless of the squark flavor content. This happens precisely since we consider only the third generation quark masses to be nonzero. Even if the masses of the first two generations are taken into account, the decay of a squark into a Higgsino and a lighter quark is highly suppressed when compared to the decay into top. Hence, finding flavor-violation with a Higgsino-like lightest gaugino is extremely challenging.

The bino limit is much more promising. Gauge invariance requires that the couplings are universal, independent of generation (in the pure bino limit). The only difference arises due to squark mixing angles. It is useful to define the flavor-violating ratio $r$

$$
r_{a} \equiv \frac{\Gamma_{\tilde{q}_{a} \rightarrow u \chi_{1}}}{\Gamma_{\tilde{q}_{a} \rightarrow t \chi_{1}}}
$$

which characterizes the amount of up-type flavor violation revealed in squark decay. To illustrate how large $r$ can be within the MRSSM parameter space, in Figs. 12 we have plotted $r$ as a function of $M_{1}$ and $\mu_{u}=\mu_{d}=\mu$ where we took $\theta_{L}=\theta_{R}=\pi / 4$ and $M_{2}=1 \mathrm{TeV}$. Maximal mixing angle implies that each squark mass eigenstate couples equally to $t$ and $u$. Taking the mass of the squark to be $500 \mathrm{GeV}$, in Figs. 12 we plot $r$ for $\tan \beta=10$.

Clearly, the region with $r \sim 1$ occurs when the lightest neutralino is mostly a bino. In addition, note that using the mixing angles shown in Eq. (2), we find that the amplitude for the 

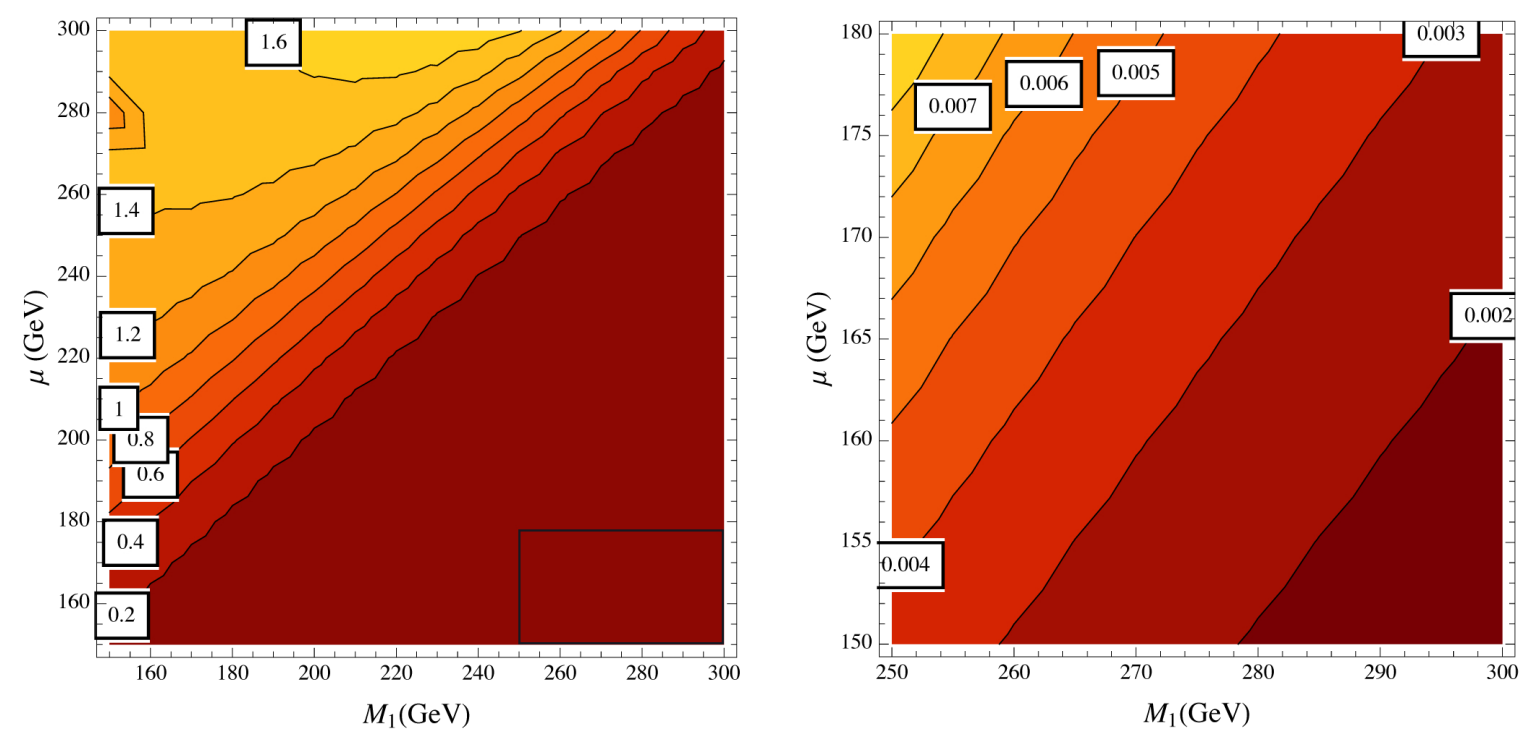

Figure 2: Contours of $r_{\tilde{u}_{R}}$ in the MRSSM for $\tan \beta=10$ and $M_{2}=1 \mathrm{TeV}$. The rightmost figure is a magnification of the rectangular region indicated in the bottom-right corner of the left figure. It shows the rapid decrease of $r$ with increasing $M_{1} / \mu$.

decay $\tilde{u}_{L_{1}}+\tilde{u}_{L_{1}}^{*} \rightarrow t+\bar{q}_{i}+\chi_{1} \bar{\chi}_{1}$ is proportional to $\sin 2 \theta_{L}$, whereas the decay of $\tilde{u}_{L_{3}}+\tilde{u}_{L_{3}}^{*}$ to the same final states is proportional to $-\sin 2 \theta_{L}$. This implies that, if these two mass eigenstates are of equal mass, then there is complete cancellation of the amplitudes (a squark GIM mechanism). Thus, in order to have a non-zero MRSSM contribution to single top, the scalar masses must be hierarchical. Finally, the gaugino hierarchy $M_{1} \ll \mu_{u}, \mu_{d}, M_{2}$ automatically ensures that all charginos and other neutralinos are also heavy. If the squarks are light enough, it is plausible that the only open channel for the two body decay of a squark is into a $\chi_{1}$ and quark.

\subsection{Signal and Background: A Quantitative Analysis}

There are considerable standard model events which constitute the background for single top events in Eq. (1) due to flavor violation. One needs to eliminate not only all the electroweak single top events but also all the standard model events which mimic single top. A quantitative study that explores the feasibility of finding flavor violation at LHC in the new physics production of single top must include all these backgrounds. In this subsection we systematically analyze signals due to sample spectra on top of the standard model backgrounds after imposing a set of cuts and show how the significance of signal vary as we change parameters in the spectrum.

We start this subsection with a description of the software tools we have used. We list all the channels that have relevant contributions towards the background along with their cross-sections after using generic parton level cuts. We then compare these background events to the signal events generated in a sample spectrum after we impose various well motivated cuts and estimate the significance of the signal. We also show how the significance varies as the spectrum itself 
is varied while keeping the same set of cuts used before. We end this subsection with a brief discussion of further refinements.

\subsubsection{Software}

To simulate the signals and background necessary for our analysis of flavor violation, we used several different Monte Carlo event generators. All events were first created at parton level utilizing matrix-element generators: ALPGENv13 [27] for the backgrounds, and MadGraphv4 [28,29] for the signal. The inputs to the matrix element generators are a set of parton level cuts, which we list under Table 1, a factorization/renormalization scale, and a parton distribution function (PDF) set. For factorization/renormalization scales, we used the default ALPGEN values when generating each background, and the squark mass $(\sim 300-500 \mathrm{GeV})$ for the signal. The default PDFs were used throughout - CTEQ5L for the background and CTEQ6L1 for the signal. All parton level events (signal + background) were passed through PYTHIA6.4 [30] for showering and hadronization, and PGS4.0 [31] for detector simulation. We use the parameters in the default pythia_card and pgs_card_ATLAS provided with MadGraph for all events 7 The primary effects of the detector simulator are:

- Limited calorimeter size and threshold: Electrons and photons are visible for $E_{T}>5 \mathrm{GeV}$, $|\eta|<3$. Muons are visible for $E_{T}>3 \mathrm{GeV},|\eta|<2.5$. Jets are visible for $E_{T}>5 \mathrm{GeV}$, $|\eta|<4$.

- Lepton energy smearing: ID efficiency for leptons in our kinematic region of interest is $80-90 \%$.

- Jet smearing: The energy/momentum for jets are smeared by an amount

$$
\frac{\delta E_{\mathrm{jet}}}{E_{\mathrm{jet}}}=\frac{0.8}{\sqrt{E_{\mathrm{jet}} / \mathrm{GeV}}} .
$$

Not only does this affect the resolution of any object reconstructed from jets, but it also affects the missing energy: $\mathbb{E}_{T}=-\sum_{\text {visible }} p_{T}$, so an error in the visible energy becomes an error in the missing energy.

- $b$-tagging: The PGS b-tag efficiency peaks around $65 \%$ with a corresponding fake rate of $\sim 1-2 \%$. Both the tag and mistag rates vary with $p_{T}, \eta$ and are modeled in PGS using a CDF fit. A $b$-jet can only be tagged if it goes through the tracker which has pseudo-rapidity extent $|\eta|<2.5$.

\subsubsection{Backgrounds}

In order to avoid potentially disastrous QCD backgrounds, we require the $W$ from top quark decay to itself decay leptonically. Therefore the signal we wish to identify, in terms of objects

\footnotetext{
${ }^{\ddagger}$ The only modification of the PGS card is a change in the sagitta resolution from $10^{-5}$ to $10^{-4}$. This primarily effects muon resolution [32].
} 
seen in the detector, is:

$$
p p \rightarrow b+j+\ell+\mathbb{E}_{T}, \ell=e, \mu
$$

An important distinction between the signal and background is the source of missing energy. The source of missing energy in the background comes from neutrinos from $W, Z$ and hadron decays, while the missing energy for the signal also contains the neutralinos. SM backgrounds for the final state given in Eq. (5) are:

- single top: $t+q \rightarrow b \ell \nu j$, where $q$ can also be $b$ quark.

- top $+W: t+W+$ jets $\rightarrow b W W+$ jets, where at least one $W$ decays leptonically.

- top pair production: both semileptonic $t \bar{t} \rightarrow b \ell \nu \bar{b} j j$ and fully leptonic $t \bar{t} \rightarrow b \bar{b} \ell \ell^{\prime} \nu \nu^{\prime}$ decay modes.

- $W+$ heavy flavor: $W+\bar{b} b \rightarrow b \bar{b} \ell \nu$.

- $Z(\bar{\nu} \nu)+$ heavy flavor: $Z+\bar{b} b \rightarrow b \bar{b}+\bar{\nu} \nu$

- $W(\ell \nu)+$ jets,

- $Z\left(\bar{\nu} \nu, \ell^{+} \ell^{-}\right)+$jets

- $W(\ell \nu)+Z(\bar{\nu} \nu)+$ jets

A few comments on the backgrounds are in order. First, many SM backgrounds contain only one neutrino and therefore only one source of missing energy. Second, several backgrounds $(t \bar{t}$ and $W+b \bar{b}$ in particular) require one of the $b$ quarks to be mistagged, while others $(W+$ jets $)$ require a light jet to fake a $b$-jet. Both of these facts will be exploited in the cuts section to separate the signal from the background. Information on the signal and backgrounds, including which generator was used and what cross section was assumed, are contained below in Table 1.

\subsubsection{A Sample Spectrum}

In order to produce signal events, we use the following MRSSM spectrum:

$$
\begin{gathered}
m_{\tilde{u}_{L_{1}}}=m_{\tilde{u}_{R_{1}}}=1 \mathrm{TeV}, \quad m_{\tilde{u}_{L_{3}}}=1 \mathrm{TeV}, \quad m_{\tilde{u}_{R_{3}}}=300 \mathrm{GeV}, \\
M_{1}=50 \mathrm{GeV}, \quad M_{2}=1 \mathrm{TeV}, \quad M_{3}=3 \mathrm{TeV}, \\
\mu_{u}=\mu_{d}=1 \mathrm{TeV}, \quad \text { and } \tan \beta=10 \\
\theta_{R}=\pi / 3, \quad \theta_{L}=0 .
\end{gathered}
$$

This is the simplest set of MRSSM parameters which has all of the attributes suggested previously for a large flavor-violating signal: the lightest neutralino is primarily a bino, there is a hierarchy between the masses of the squarks which mix among themselves, and all squarks are heavy enough so that the decay channel to the LSP + top is open. 


\begin{tabular}{|c|c|c|}
\hline Process & $\sigma$ & \# events \\
\hline \hline$\tilde{u}_{i} \tilde{u}_{i}^{*} \rightarrow t j \chi_{1} \bar{\chi}_{1} \rightarrow \ell b j \nu \chi_{1} \bar{\chi}_{1}$ & $1.88 \mathrm{pb}$ & 14,300 \\
\hline \hline$t \bar{t} \rightarrow b \bar{b} j \ell \nu$ & & \\
$t \bar{t} \rightarrow b \bar{b} \ell \ell^{\prime} \nu \nu^{\prime}$ & $197 \mathrm{pb}$ & $2.90 \times 10^{6}$ \\
$t+q \rightarrow b j \ell \nu$ & $49.1 \mathrm{pb}$ & $2.09 \times 10^{6}$ \\
$t+b \rightarrow b b \ell \nu$ & $59.2 \mathrm{pb}$ & $1.8 \times 10^{6}$ \\
$t($ inc. $)+W(\ell \nu)$ & $2.28 \mathrm{pb}$ & $1.12 \times 10^{6}$ \\
$t($ inc. $)+W(\ell \nu)+j$ & $31.3 \mathrm{pb}$ & $1.01 \times 10^{6}$ \\
$W+\bar{b} b \rightarrow \bar{b} b \ell \nu$ & $17.6 \mathrm{pb}$ & $8.75 \times 10^{5}$ \\
$Z+\bar{b} b \rightarrow \bar{b} b \bar{\nu} \nu$ & $24.7 \mathrm{pb}$ & $6.30 \times 10^{5}$ \\
$W Z j j \rightarrow \ell+3 \nu+j j$ & $1.23 \mathrm{pb}$ & $2.1 \times 10^{5}$ \\
$W+$ jets $\rightarrow \ell \nu+$ jets & & \\
$W+j$ & $525.3 \mathrm{pb}$ & $1.21 \times 10^{6}$ \\
$W+j j$ & $744.5 \mathrm{pb}$ & $6.40 \times 10^{6}$ \\
$W+$ more than $2 j$ & $396 \mathrm{pb}$ & $3.58 \times 10^{6}$ \\
$Z+$ jets $\rightarrow \ell^{+} \ell^{-}+$jets & & \\
$Z+j$ & $1737 \mathrm{pb}$ & $1.27 \times 10^{7}$ \\
$Z+j j$ & $967 \mathrm{pb}$ & $6.4 \times 10^{6}$ \\
$Z+$ more than $2 j$ & $291 \mathrm{pb}$ & $2.9 \times 10^{6}$ \\
$Z+$ jets $\rightarrow \bar{\nu} \nu+$ jets & & \\
$Z+j$ & $79 \mathrm{pb}$ & $3.49 \times 10^{6}$ \\
$Z+j j$ & $439 \mathrm{pb}$ & $4.24 \times 10^{6}$ \\
$Z+\operatorname{more}$ than $2 j$ & $229 \mathrm{pb}$ & $2.89 \times 10^{6}$ \\
\hline
\end{tabular}

Table 1: Signals and background processes considered. Cross sections include the branching ratios to leptons $(\ell=\mu, e)$. All events were generated with parton level cuts of $p_{T, j}, p_{T, l}>$ $15.0 \mathrm{GeV},\left|\eta_{j}\right|,\left|\eta_{b}\right|<4.0$, and $\Delta R_{j j}>0.4$, while the $W+$ jets and $Z(\bar{\nu} \nu)+$ jets backgrounds were generated with the additional cut of $\mathbb{E}_{T}>75.0 \mathrm{GeV}$. Jet-parton matching, following the MLM scheme [33] was used in all backgrounds except for single-top, where it is not yet available, and $W / Z+$ more than 2 jets. The parton level cuts were imposed to make event generation more efficient; ideally parton level cuts should be softer then the analysis cuts to allow initial and final state radiation to have an effect. We used the default factorization and renormalization schemes/scales for all of the above events. The number of events for the background was chosen to roughly correspond to the number of events expected (after the full analysis) after $10 \mathrm{fb}^{-1}$ of integrated luminosity.

The gluino and all squarks except one right-handed up-type squark, $\tilde{u}_{R_{3}}$ are heavy, thus $\tilde{u}_{R_{3}}$ completely dominates SUSY production at the LHC. We chose $\tilde{u}_{R_{3}}$ to be light (rather than $\tilde{u}_{L_{3}}$ ) as it is an electroweak singlet, so its mass can be adjusted without affecting the other states in the spectrum. Since $\mu_{d}, \mu_{d}, M_{2}>m_{\tilde{u}_{R_{3}}}$, all the charginos and all the neutralinos except the LSP are heavier than the $\tilde{u}_{R_{3}}$ squark. The only open two-body decay channels are 
$\tilde{u}_{R_{3}} \rightarrow \chi_{1}+t, \tilde{u}_{R_{3}} \rightarrow \chi_{1}+u$ both of which involve the weak bino coupling, hence $\tilde{u}_{R_{3}}$ is very narrow, $\Gamma_{\tilde{\chi}} \sim \mathcal{O}(1 \mathrm{GeV})$. By restricting the $\tilde{u}_{R_{3}}$ decays to $q+\tilde{\chi}_{1}$ we enhance the cross section for the flavor-violating signal, however this comes at a price: we have fewer tools to fight the SM background, and our signal cannot provide much information on the details of the MRSSM spectrum. To get detailed spectrum information a scenario which has smaller cross section but contains long SUSY decay chains, similar to those considered in Ref. [34-38], would likely be better.

\subsubsection{Cuts}

To suppress the SM background we impose the following cuts:

- Exactly 1 lepton, $p_{T}>30 \mathrm{GeV},|\eta|<2.5$. This serves as our primary trigger and suppresses QCD backgrounds.

- Exactly 2 jets, within $|\eta|<3.5$. One jet must be tagged as a $b$-jet and have $p_{T}>50 \mathrm{GeV}$, while the untagged jet must have $p_{T}>80 \mathrm{GeV}$.

- $\mathbb{E}_{T}>100 \mathrm{GeV}$

- $\mathbb{E}_{T}>0.25 \times M_{e f f}$, where $M_{e f f}=\sum_{j, \ell} p_{T}+\mathbb{E}_{T}$

- Transverse mass of the $W, m_{T, W}>120 \mathrm{GeV}$

These cuts were not optimized in any rigorous way.

The motivation for these cuts is the following: First, for a process where the sole source of (true) missing energy is the neutrino from $W$ decay, the transverse mass of the lepton- $\mathbb{E}_{T}$ system $\left(m_{T, W}=\sqrt{\left(E_{\ell}+\mathbb{E}_{T}\right)^{2}-\left(p_{T, \ell}+p_{T, \nu}\right)^{2}}\right)$ exhibits a sharp edge at $M_{W}$, at least up to detector resolution effects. If there are multiple sources of missing energy, as in the MRSSM flavor-violating signal, the transverse mass distribution is much smoother. Thus, by cutting on $m_{T, W} \gg M_{W}$, we strongly suppress all backgrounds except fully leptonic $t \bar{t}, t W(2 \ell 2 \nu b), Z(\bar{\nu} \nu)+$ $\bar{b} b$, and $W Z(3 \ell+\nu)+$ jets. Of the remaining backgrounds, fully leptonic $t \bar{t}$ is the largest, despite the fact that it matches the signal only when one of the leptons is missed or falls outside the calorimeter. To reduce the residual leptonic $t \bar{t}$ background, we exploit the fact that the $\mathbb{E}_{T}$ and non b-jet $p_{T}$ are typically higher for the signal than for the background. The jet $p_{T}$ is higher for the signal since it arises from a massive squark decaying to two essentially massless objects. The heavier the squark, the harder this jet will be. The missing energy is also larger for the signal simply because there are more sources - two neutralinos and a neutrino for the signal, compared to just two neutrinos for the background. The optimum value for the $\mathbb{E}_{T}$ cut also depends on the mass of the squark, with more massive squarks able to deposit more missing energy.

The other cut, exactly two jets, is designed to remove the remaining semileptonic $t \bar{t}$ and $W / Z+$ jets backgrounds. These processes have such large cross sections that they pollute our signal if we only impose the $m_{T, W}$ cut. Also, by adding this cut we eliminate several MRSSM 
backgrounds. The more jets we allow, the more MRSSM processes can contribute, obscuring the interpretation of an excess as flavor violating new physics. The signal and background efficiencies under these final cuts, and the number of events generated in $10 \mathrm{fb}^{-1}$ of integrated luminosity are summarized in Table 2 .

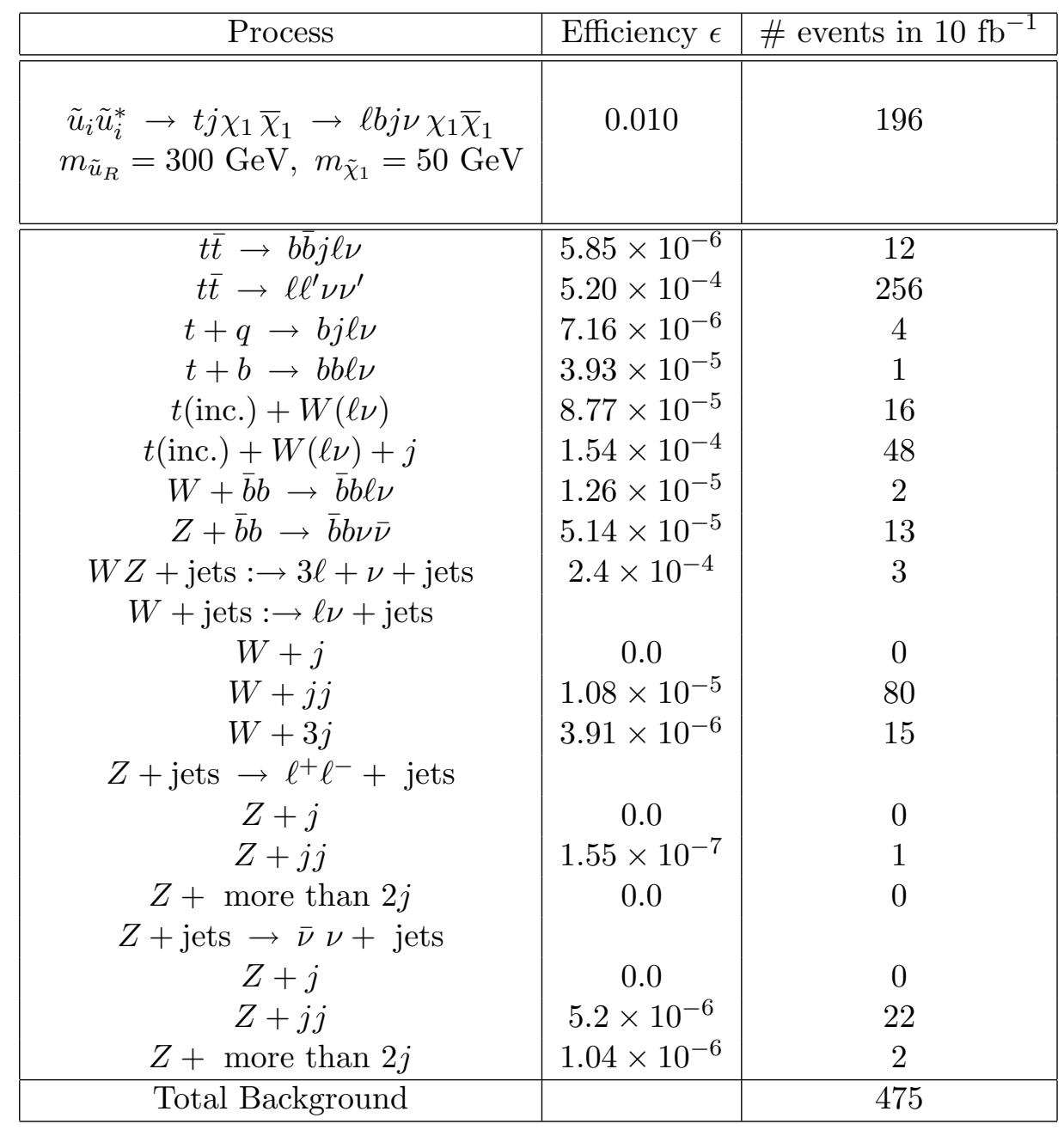

Table 2: Cut efficiency and number of post-cut events assuming an integrated luminosity of $10 \mathrm{fb}^{-1}$.

Weighting the generated events by their respective cross sections, we combined the signal and background histograms for several variables. We show the results in Fig. 3.

\subsubsection{Signal Significance as the Spectrum Varies}

Up to now we have identified a set of cuts that separates background from signal using a particular sparticle spectrum. Here we show that the same set of cuts can also separate signal from background over a wider range of model parameters. We characterize our ability to find 

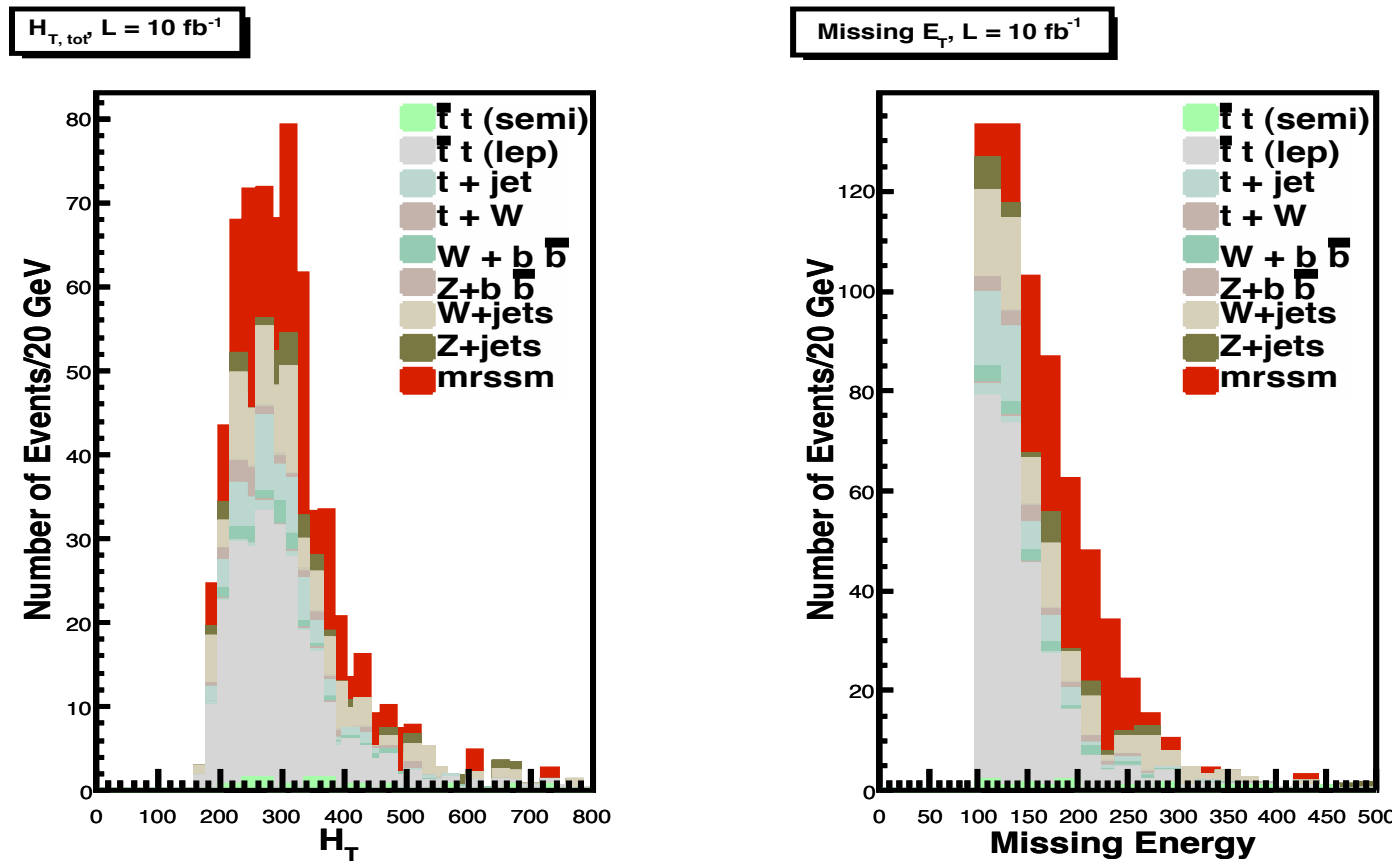

Figure 3: Number of events as a function of $H_{T}$ (left figure) and $\mathbb{E}_{T}$ (right figure) assuming an integrated luminosity of $10 \mathrm{fb}^{-1}$ at $14 \mathrm{TeV}$. The MRSSM signal is for the spectrum given in Sec. 3.2 .3 .

the signal over background using "significance",

$$
\mathcal{S}=\frac{S}{\sqrt{S+B}}
$$

where $S$ is the number of signal events and $B$ is the number of background events.

Using the same set of cuts listed in Sec. 3.2.4, we have calculated the significance of the signal spanning over a grid in $m_{\tilde{u}_{R_{1}}}$ and $M_{1}$. We held the following parameters constant

$$
\begin{gathered}
m_{\tilde{u}_{L_{1}}}=1 \mathrm{TeV}, \quad m_{\tilde{u}_{L_{3}}}=m_{\tilde{u}_{R_{3}}}=1 \mathrm{TeV} \\
M_{2}=1 \mathrm{TeV}, \quad M_{3}=3 \mathrm{TeV}, \quad \mu_{u}=\mu_{d}=1 \mathrm{TeV}, \\
\sin \theta_{L}=0 \text { and } \tan \beta=10
\end{gathered}
$$

Our result is shown in Fig. 4, where we have interpolated the grid to form the contours of $\mathcal{S}$ as shown. Even without optimizing the cuts, we see that the flavor-violating significance exceeds 1 for squarks up to about $500 \mathrm{GeV}$ and neutralino LSP masses up to about $250 \mathrm{GeV}$. Further optimization, suitable for specific collider detector characteristics, can surely probe even larger regions of parameter space or increase the significance at any given spectrum point.

When $\theta_{R}=\pi / 4$ the mass eigenstate squark is an equal mixture of top and up squarks, so one might expect that the largest single-top signal comes in this scenario. However, as we can see from Fig. 4 this depends on the mass of the squark. When the squark mass is comparable to 


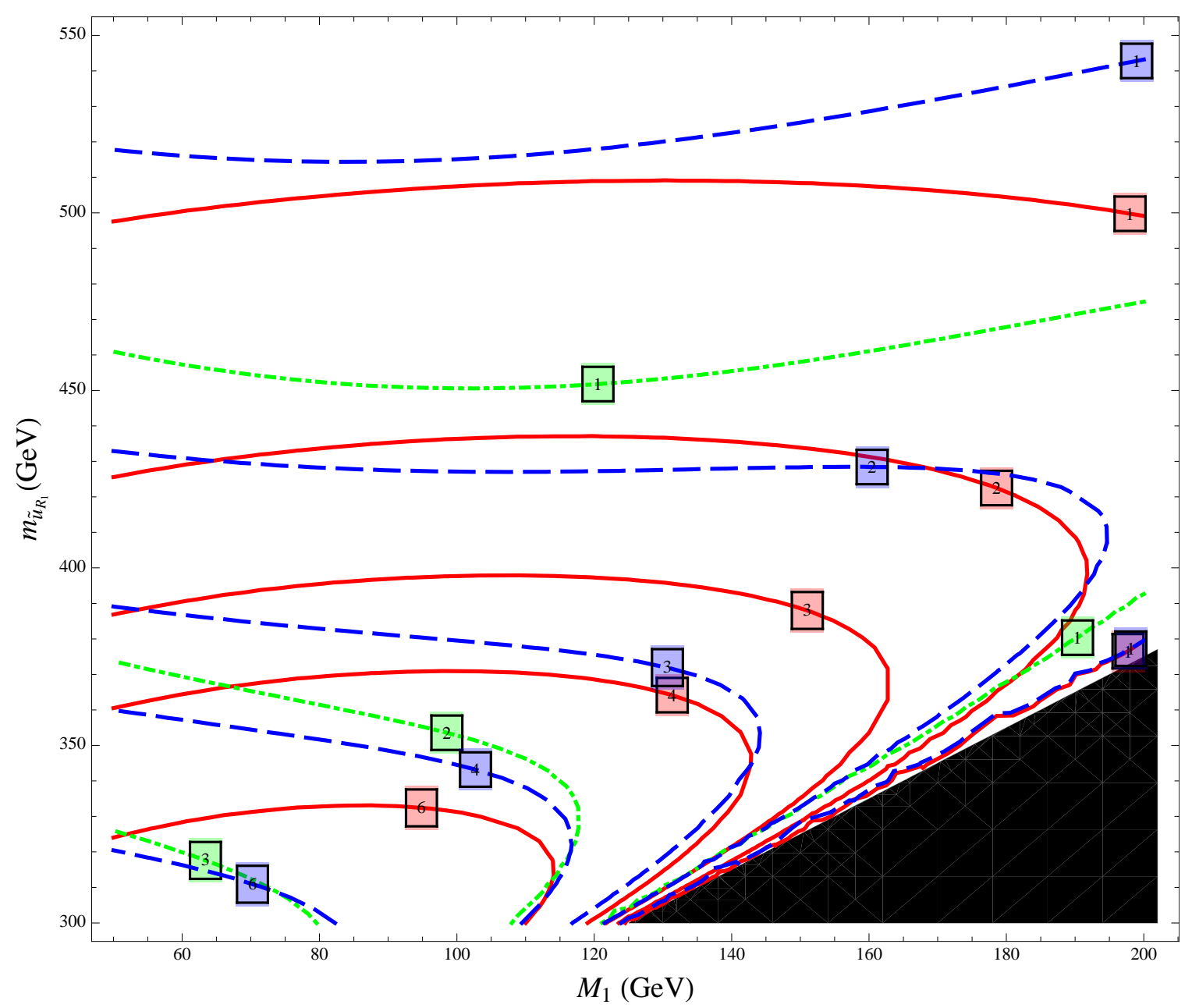

Figure 4: Significance $\mathcal{S}$ of the signal assuming an integrated luminosity of $30 \mathrm{fb}^{-1}$ at $14 \mathrm{TeV}$. In order to produce this figure we have assumed the spectrum shown in Eq. (3.2.5). Red (solid) contours are for $\theta_{R}=\pi / 3$, blue (dashed) contours corresponds to $\theta_{R}=\pi / 4$ and finally green (dot-dashed) contours are due to $\theta_{R}=\pi / 6$. The channels responsible for background events are listed in Table. 1 and we use the same set of cuts as described in the subsection. 3.2.4. In the black region on the bottom right corner the neutralino is too heavy and a single top event is kinematically inaccessible.

the top quark mass, decays $\tilde{u} \rightarrow t+\chi_{1}$ are kinematically suppressed and the overall single-top signal suffers. For larger mixing angles (like $\theta_{R}=\pi / 3$ ), the mass eigenstate squark is more top-squark than up-squark. This offsets the kinematic suppression at low squark mass and leads to a larger single-top signal. 


\subsubsection{Further Refinements}

The analysis in the last section concentrated on eliminating the SM backgrounds, however there will certainly be additional supersymmetric contributions to the $b+\ell+j+\mathbb{E}_{T}$ signal which we would like to eliminate. These supersymmetric backgrounds are more difficult to gauge without knowing the details of the complete spectrum. However, one process which will surely contribute as a background is $p p \rightarrow \tilde{q} \tilde{q}^{*} \rightarrow t \bar{t}$, where one of the $b$ quarks goes untagged. In fact, the correlation between the $p p \rightarrow \tilde{q} \tilde{q}^{*} \rightarrow t \bar{t}$ and the $p p \rightarrow \tilde{q} \tilde{q}^{*} \rightarrow t+$ jet is crucial to isolate the flavor-violating physics. Any enhancement to $t \bar{t}$, even in the absence of flavor violation - such as $p p \rightarrow \tilde{q} \tilde{q}^{*} \rightarrow t \bar{t}$ in the flavor-blind MSSM - will lead to an excess in the final state signal we examined here coming from missed or mistagged $b$-jets. However, flavor violation will lead to a decrease in the $t \bar{t}$ signal and an increase in the single-top signal relative to a flavor-blind model. Consider a scenario where the $\tilde{q} \tilde{q}^{*}$ cross section is $\sigma$, and let us take just one mixing angle and neglect the mistag rate which affects all channels identically. Squark pair production in a flavor-blind model (i.e., top squark production) leads to all events in $t \bar{t}$ and none in single top. Conversely, squark pair production in a model with squark flavor violation will generate $\sigma \cos ^{4} \theta$ in $t \bar{t}$ and $\sigma \cos ^{2} \theta \sin ^{2} \theta$ in single top, assuming the kinematics of the process is held fixed as the mixing angle is changed. In practice, the comparison is more difficult due to differences in the analysis cuts applied and multiple squark mixing angles.

In the SM, an asymmetry in the charge of the final state lepton can help improve the singletop significance [39], however this is not the case for the MRSSM flavor-violating signal. The lepton asymmetry for SM single-top due to larger $u, d$ PDFs compared to $\bar{u}, \bar{d}$. The dominant parton-level contribution to our signal is $g g \rightarrow \tilde{u}_{R} \tilde{u}_{R}^{*}$, so we are insensitive to the quark PDFs. The final state squark and anti-squark are equally likely to decay to a top (anti-top), thus we expect an equal number of positively and negatively charged leptons.

There are several ways in which one could improve upon the analysis presented here. First there are more sophisticated optimization techniques (neural net, decision trees, etc.) which can extract the correlations between the observables better than we can do by hand. Second, we can use the fact that our signal has only one $b$-quark, while the dominant background (both SM and supersymmetric) has two. One way to take advantage of this would be to use a $b$-tagging scheme which places a premium on getting the $b$ 's tagged correctly. While the analysis presented here uses the default PGS $b$-tag parametrization, which has a tag rate of $\sim 50 \%$ and a mistag rate of $1-2 \%$, identification rates as high as $90 \%$ are possible [42]. High tag rates have correspondingly high fake rates $\sim 30 \%$, however the backgrounds to our flavor-violating signal which come from fake $b$-jets are all highly suppressed by the $m_{T, W}$ cut. Thus we could hopefully reduce all $t \bar{t}$ backgrounds without causing a huge enhancement in backgrounds such as $W+$ jets, $W+b \bar{b}$. A dedicated study of the viability of this improved b-tag technique is, however, beyond the scope of this paper. 


\section{Case II: A neutralino NLSP and Gravitino LSP}

We now turn to considering squark flavor violation when the neutralino decays within the detector and part of the neutralino energy can be measured by detecting visible particles that result out of the neutralino decay.

In this scenario the gravitino is the LSP, which escapes the detector as missing energy. As before, we are interested in the pair production of squarks which decay to quarks of different flavors and the lightest neutralinos. The new feature is that the neutralinos decay to the gravitino plus a photon $/ Z /$ neutral Higgs.

\subsection{Neutralino NLSP Decay Models}

The two-body decay width of a neutralino into the gravitino and a spectator particle $(X)$ is simply given as [43]:

$$
\Gamma(\tilde{\chi} \rightarrow \tilde{G}+X)=\frac{\kappa m_{\tilde{\chi}}^{5}}{96 \pi M_{\mathrm{pl}}^{2} \tilde{m}_{3 / 2}^{2}}\left(1-\frac{m_{X}^{2}}{m_{\tilde{\chi}}^{2}}\right)^{4},
$$

where $\kappa$ is an order one mixing angle. The range of NLSP masses that we consider is typically comparable to $Z$ or Higgs mass, and thus decays to the $Z$ or $h$ are kinematically suppressed compared to the decays to a photon. For example, when $\kappa_{Z \tilde{G}} \approx \kappa_{\gamma \tilde{G}}$, we find $\Gamma_{\gamma \tilde{G}} / \Gamma_{Z \tilde{G}} \approx 0.16$ for a $150 \mathrm{GeV}$ NLSP. Three-body decays through an off-shell $Z$ or Higgs are suppressed even further. The only exceptions happen when $\kappa_{\gamma \tilde{G}} \ll \kappa_{Z \tilde{G}}$, which occurs when the NLSP is Higgsino-like, i.e., $\mu_{u}$ and/or $\mu_{d} \ll M_{1}, M_{2}$. A Higgsino NLSP, however, leads to a substantial suppression of the flavor-violating signal, due to the large top quark Yukawa coupling. This is clearly evident by looking at the rightmost figures in Figs. 12. We will see that this seriously impacts the prospects for finding flavor-violation with a chargino NLSP in Sec. 5. In the case of a Higgsinolike neutralino NLSP, substantial suppression is also expected. It would take a dramatic signal, such as a moderate lifetime neutralino NLSP decaying into a $Z$ with significant measurable impact parameter, to find evidence of squark flavor-violation in this case.

\subsection{Setup and Feasibility}

Given the discussion above, the remainder of Case II focuses on neutralino NLSP decay to a photon. The flavor-violating signal arises from:

$$
\begin{aligned}
p+p \rightarrow & \left\{\begin{array}{c}
\tilde{u}_{L_{a}}+\tilde{u}_{L_{a}}^{*} \\
\tilde{u}_{R_{a}}+\tilde{u}_{R_{a}}^{*}
\end{array}\right\} \rightarrow \text { top }+ \text { jet }+\chi_{1}+\bar{\chi}_{1} \\
& \rightarrow W+\text { b jet }+ \text { jet }+2 \text { photons }+2 \text { gravitinos } \\
p+p \rightarrow & \left\{\begin{array}{c}
\tilde{d}_{L_{a}}+\tilde{d}_{L_{a}}^{*} \\
\tilde{d}_{R_{a}}+\tilde{d}_{R_{a}}^{*}
\end{array}\right\} \rightarrow \text { b jet }+ \text { jet }+\chi_{1}+\bar{\chi}_{1} \\
& \rightarrow \quad \text { b jet }+ \text { jet }+2 \text { photons }+2 \text { gravitinos } .
\end{aligned}
$$


Missing energy comparison

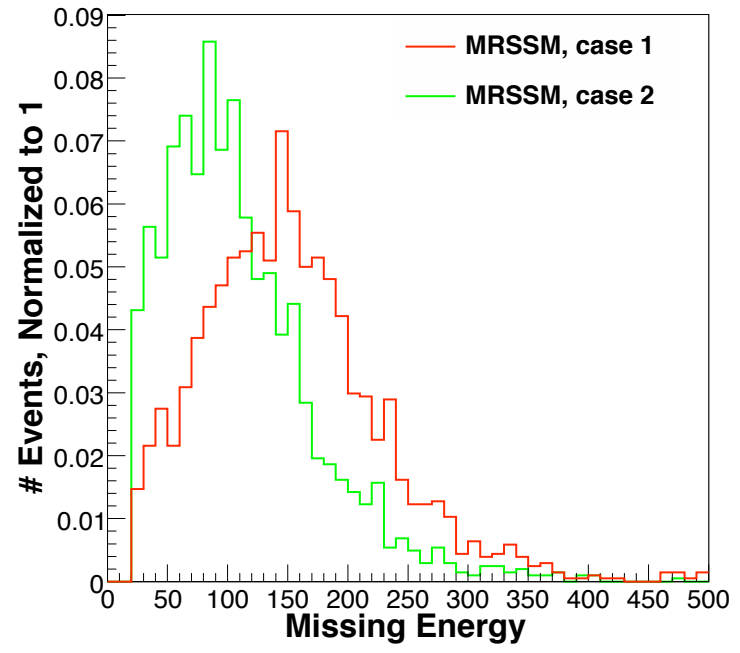

Figure 5: Difference in $\mathbb{E}_{T}$ in MRSSM signals in Case I and II. In Case II, we took $m_{\tilde{G}}=$ $1 \mathrm{eV}$. For comparison purposes, only soft cuts were imposed: $\geq 2$ jets, $p_{T}>20 \mathrm{GeV}, \mathbb{E}_{T}>$ $20 \mathrm{GeV}, 1$ lepton, $p_{T}>25 \mathrm{GeV}$.

The signal consists of two hard photons, one $b$ jet and a jet of any other flavor. In the case of up-type squarks we look for an additional lepton that results from the leptonic decay of the top. The neutrino and gravitinos constitute the missing energy $\mathbb{E}_{T}$. However, as mentioned before, we expect that the missing energy is less whenever the neutralino decays inside the detector, since part of the neutralino energy/momentum is redeposited in the detector in the form of (visible) photons. This can easily be verified by comparing missing energy distributions for identical spectrum in Cases I and II as shown in Fig. 5.

Standard model backgrounds with two hard photons are relatively rare, making this scenario of flavor violation nearly free of SM backgrounds. Exactly what information can be gleaned from the flavor violating signal depends on the characteristic decay length of $\tilde{\chi}_{1}$ :

$$
L=34 \kappa^{-1}\left(E^{2} / m_{\tilde{\chi}_{1}}^{2}-1\right)^{1 / 2}\left(\frac{100 \mathrm{GeV}}{m_{\tilde{\chi}_{1}}}\right)^{5}\left(\frac{\tilde{m}_{3 / 2}}{1 \mathrm{eV}}\right)^{2} \mu \mathrm{m}
$$

where $E$ is the energy of the NLSP in the lab frame.

For prompt neutralino decays, the two photons from the processes shown in Eqs. (9 10) point back to the primary vertex. While there is no displaced vertex, the relatively high transverse momenta $\left(p_{T}\right)$ of these prompt photons is still highly effective for background rejection. Note that, since the photons provide the trigger and excellent background rejection, one can extend this study to find flavor-violating squark mixing resulting from the down type squark mass matrix, which was not possible in Case I.

If the neutralino is long-lived, while still decaying before it enters the electromagnetic calorimeter, the signal becomes much more interesting but also somewhat more complicated. 
Because of the finite opening angle between the resulting photon and the gravitino, the photon may not point back to the primary interaction point. The identification of a non-pointing photon has several advantages, since it makes the signal virtually free of Standard Model background. However, non-pointing photons may enter the calorimeter at a significant angle, causing the electromagnetic shower to be spread out over a larger number of cells making the shower shapes wider and leading to losses in reconstruction and efficiency. Detailed search strategies and efficiencies for the non-pointing photons in ATLAS can be found in [40-42]. The electromagnetic calorimeter can be used not only to measure the direction and the time of the electromagnetic shower, but also to determine the mean lifetime of the neutralino. As long as the neutralino decays before the calorimeter, both $\eta$ and $\phi$ can be measured and a vector corresponding to the path of the photon can be constructed. Although the exact decay point cannot be measured, the path of the photon can be extrapolated to the beam axis, and further information can be extracted from the the distance between this point and the primary vertex. Also photons produced from long-lived neutralinos arrive later than the ones directly from the primary vertex. Calorimeter timing information can be used to gather further information about the lifetime of the neutralino.

If neutralinos are sufficiently long-lived so that they decay well outside the detector, the resulting photons will be missed. This "collider-equivalent" LSP leads to a collider signal that is exactly the same as that studied in Case I (see Sec. 3).

\subsection{Sample Signal with Background}

Having identified the different scenarios within Case II, we now examine the signals and background for a simple subset of the parameter space. We use the same spectrum parameters as in Case I: all superpartners are heavy $(\geq 1 \mathrm{TeV})$ except for one neutralino with mass $m_{\tilde{\chi}_{1}}=50 \mathrm{GeV}-200 \mathrm{GeV}$, and one right handed up-type squark, $m_{\tilde{u}_{R}}=300-500 \mathrm{GeV}$. The spectrum parameters must be supplemented with a gravitino mass which we take to be $1 \mathrm{eV}$. We are free to make this choice independent of the other soft masses, and it leads to a neutralino lifetime of order few nanoseconds and thus a prompt decay. The restriction to prompt decays is necessary as our software tools are inadequate to accurately depict scenarios with displaced vertices. The signal events we look for consist of $\ell+b+$ jet $+\gamma \gamma+\mathbb{E}_{T}$.

\subsubsection{Backgrounds}

The primary SM backgrounds for this case are the same as in Case I, however we are now interested in the fraction of events which contain final state photons. Photons appear in the final state of these backgrounds due to bremsstrahlung, from hadron decay products, or from jets faking photons. The extra photons require a price of $\alpha_{e m}$ if they are real, or a factor of the jet-photon fake rate if the photons are actually jets faking photons. Real photons emitted as bremsstrahlung are usually soft, while photons from subsequent hadron decays are usually

buried within a jet. The jet-photon fake rate is $p_{T}$ and $|\eta|$ dependent and is expected to be 


\begin{tabular}{|c|c|}
\hline Process & \# events in $10 \mathrm{fb}^{-1}$ \\
\hline \hline & \\
$\tilde{u}_{R, i} \tilde{u}_{R, i}^{*} \rightarrow t j \chi_{1} \bar{\chi}_{1} \rightarrow \ell b j \gamma \gamma+\mathbb{E}_{T}$ & 481 \\
$m_{\tilde{u}_{R}}=300 \mathrm{GeV}, m_{\tilde{\chi}_{1}}=50 \mathrm{GeV}$ & \\
\hline \hline$t \bar{t} \rightarrow b \bar{b} j \ell \nu$ & 1.3 \\
$t \bar{t} \rightarrow \ell \ell^{\prime} \nu \nu^{\prime}$ & 1.4 \\
$t+q \rightarrow b j \ell \nu$ & 0 \\
$t+b \rightarrow b b \ell \nu$ & 0 \\
$t($ inc. $)+W(\ell \nu)$ & 0 \\
$t($ inc. $)+W(\ell \nu)+j$ & $\leq 1$ \\
$W+\bar{b} b \rightarrow \bar{b} b \ell \nu$ & 0 \\
$Z+\bar{b} b \rightarrow \bar{b} b \nu \bar{\nu}$ & 0 \\
$W Z+$ jets $\rightarrow 3 \ell+\nu+$ jets & 0 \\
$W(\ell \nu)+$ jets & $\leq 1$ \\
$Z\left(\ell^{+} \ell^{-}\right)+$jets & 0 \\
$Z(\bar{\nu} \nu)+$ jets & 0 \\
\hline Total Background & $\leq 5$ \\
\hline
\end{tabular}

Table 3: Number of post-cut events assuming an integrated luminosity of $10 \mathrm{fb}^{-1}$. The same cross sections and event samples were used as in Sec. 3. Zero events indicates no events in the generated data sample passed the cuts, while $\leq 1$ indicates some events did pass the cuts, but, once properly normalized, the number of events was some fraction less than 0.5 . The $W / Z+$ jets entries are the sum of the of the $+j,+2 j$, and + more than $2 j$ backgrounds. Because the cut on the transverse mass of the $W$ is no longer needed to suppress SM background, the number of signal events in this case is substantially larger than the corresponding point in Case I.

$\sim 0.1-0.01 \%[24,40]$. Furthermore, the $p_{T}$ spectrum of the fake jets depends on how one models faking. Since the fake rate is model dependent and sufficiently less than $\alpha_{e m}$, we will not consider faked photons in this work .

The signal, by contrast, results from a neutralino decaying to a gravitino plus photon. For practical purposes, the branching ratio is $\mathcal{O}(1)$. Moreover, the photons from the signal can be hard, $p_{T, \gamma} \sim m_{\chi_{1}} / 2$. Thus, by simply requiring the presence of final state photons, we expect all SM backgrounds can be substantially reduced compared to their counterparts in Case I.

\subsubsection{Cuts}

From the above argument, we expect the combination of hard photons with typical SUSY features (large $\mathbb{E}_{T}$, many high $p_{T}$ objects) has little SM background. This was verified in a recent ATLAS study of GMSB scenarios [42]. Specifically, in Ref. [42] the $W / Z+$ jets and $t \bar{t}+$ jets backgrounds were essentially eliminated by requiring two hard $\left(p_{T}>20 \mathrm{GeV}\right)$ photons on top of conventional SUSY cuts $\left(\geq 4\right.$ jets, $p_{T}>50 \mathrm{GeV}$, leading jet $p_{T}>100 \mathrm{GeV}, \mathbb{E}_{T}>100 \mathrm{GeV}$, 


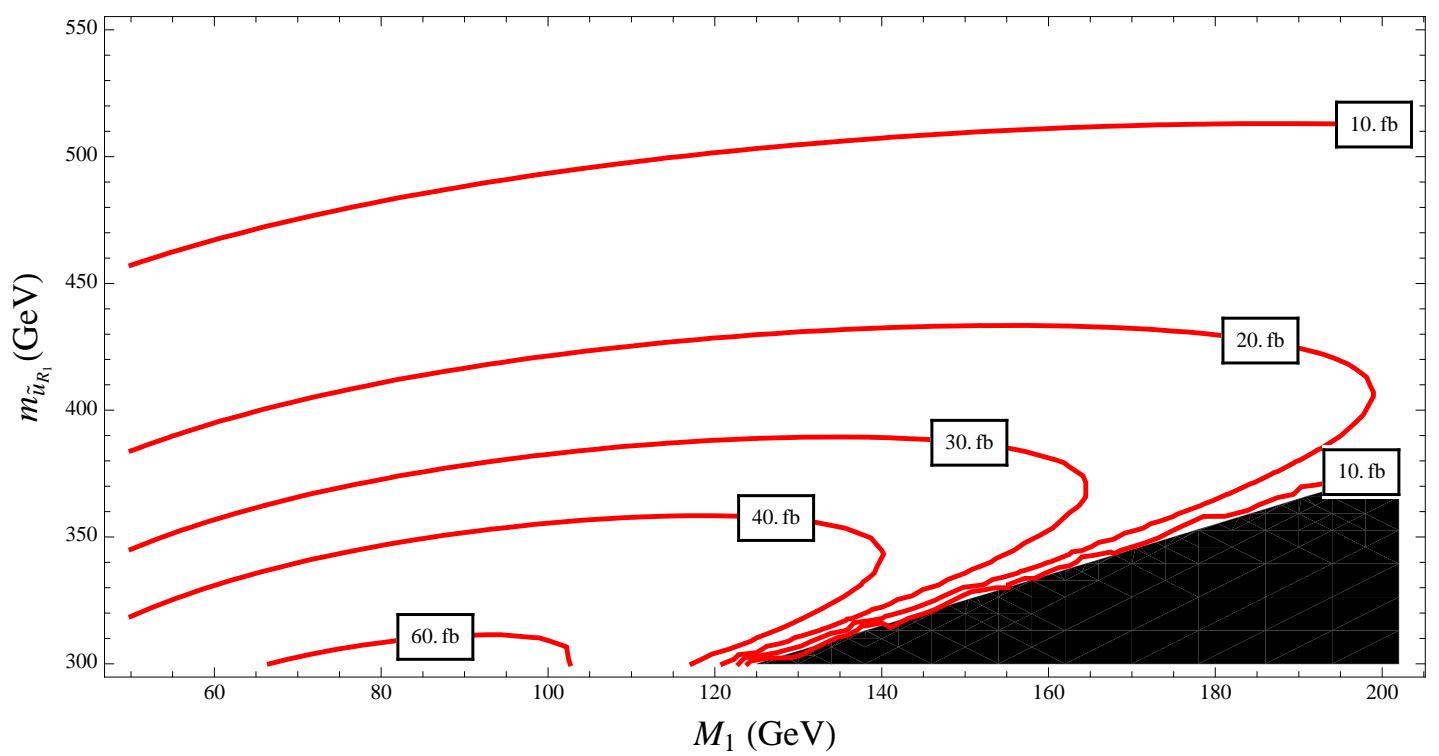

Figure 6: Signal cross section as a function of $\tilde{q}_{R}$ mass and $\chi_{1}$ mass, assuming $\theta_{R}=\pi / 3$. As before, the area in the bottom right is kinematically forbidden.

$\left.\mathbb{E}_{T}>0.2 \times M_{\text {eff }}\right)$. However, the ATLAS study only looked at discovery potential for benchmark GMSB points, while we are looking to pick out a flavor violation signal and therefore we need to tailor the cuts beyond what was done in Ref. [42]. First, we require a single b-tag and a lepton. Second, because we considered the shortest possible signal decay chains, the number of jets in the signal after showering and hadronization is less than in a typical GMSB benchmark point. To account for this we reduce the minimum number of jets required in the signal to 2 . Since we have adjusted the cuts, and are looking for events with tagged b-jets, we have included the backgrounds $W+\bar{b} b+$ jets, $Z+\bar{b} b+$ jets, $t+\{q, b, W\}+$ jets as well as the backgrounds studied in Ref. [42]. The final cuts we impose in Case II are thus:

- Exactly two photons, $p_{T}>20 \mathrm{GeV},|\eta|<2.5$. These photons serve as our primary trigger.

- Exactly one lepton $(\ell=e, \mu)$ with $p_{T}>30 \mathrm{GeV},|\eta|<2.5$.

- $\geq 2$ jets, $p_{T}>50 \mathrm{GeV}$. The leading jet must have $p_{T}>100 \mathrm{GeV}$ and exactly one jet must be tagged as a b-jet.

- $\mathbb{E}_{T}>100 \mathrm{GeV}$.

- $\mathbb{E}_{T}>0.2 \times M_{e f f}$, where $M_{e f f}=\sum_{i=j, \ell, \gamma} p_{T, i}+\mathbb{E}_{T}$.

Although we have changed the cuts from Ref. [42], we reach the same conclusion: the requirement of two hard photons renders all SM backgrounds to be negligible. To be explicit, the number of backgrounds events, assuming $10 \mathrm{fb}^{-1}$ of integrated luminosity and after imposing the above cuts is shown in Table 3. A sample signal point, with $m_{\tilde{u}_{R}}=300 \mathrm{GeV}, m_{\tilde{\chi}_{1}}=50 \mathrm{GeV}$, is also shown. 
With virtually no SM backgrounds, a flavor violation signal might be extracted from just a handful of events. Of course, as in Case I, some supersymmetric backgrounds will survive the cuts above - but these are difficult to quantify. To demonstrate the order of magnitude signals one can expect in this scenario, we therefore show in Fig. 6 the MRSSM signal cross section after imposing the cuts above in a sample region of parameter space. Hundreds of background-free flavor-violating events are expected in the region shown given just $10 \mathrm{fb}^{-1}$ of data.

\section{Case III: A Chargino NLSP and Gravitino LSP}

Finally we discuss the scenario with a chargino NLSP and a gravitino LSP. Such a scenario does not ordinarily occur in the MSSM. In the MRSSM it is quite generic [44]. Given the fact that we only consider large $M_{2}$, the lightest chargino lighter than a neutralino can only occur when one or both of $\mu_{u}, \mu_{d}$ is much smaller than $M_{1}$. That necessarily implies that the NLSP is mostly one of the charged Higgsinos.

Just like in the last section, the decay length of the chargino is crucial. Since LEP II has ruled out charginos lighter than about $100 \mathrm{GeV}[45]$, the 2-body decay $\chi_{1}^{ \pm} \rightarrow W^{ \pm} \tilde{G}$ is open, but the width is suppressed by the small wino content of the chargino. The 2-body decay $\chi_{1}^{ \pm} \rightarrow H^{ \pm} \tilde{G}$ may also be open, or, if the charged Higgs is heavier than the lightest chargino, the decay may proceed through a 3-body decay with an off-shell $H^{ \pm}$. Exactly which decay dominates is parameter-dependent. Decays to a $W$ have the advantage of the nontrivial branching fraction of the $W$ into $e$ and $\mu$. The decay into or through a charged Higgs $H^{+}$could result in $t \bar{b}$ (if open), or $\tau^{+} \nu_{\tau}$ or $c \bar{s}$ [45]. The leptonic branching fraction of a chargino NLSP, therefore, may be rather suppressed.

A larger problem lies in the intrinsic suppression of the flavor-violating decays. Higgsinos mostly couple to the third generation quarks. Even when the mixing angle in the squark mass matrix is maximal, the large third generation Yukawa couplings and small gaugino content imply that the squarks overwhelmingly decay to the quarks of third generation. This makes it extremely challenging to generate a flavor-violating signal through squark decay.

If the decay length of the chargino (in this case a Higgsino) is large enough so that isolated charged tracks of the chargino NLSPs are visible, this dramatic signal may readily be found. Certainly a clearly visible isolated track will significantly reduce (or potentially eliminate) standard model backgrounds [41,46-49]. Below we list the decay chain we look for (chargino decays are not shown):

$$
\begin{aligned}
p+p \rightarrow & \left\{\begin{array}{l}
\tilde{u}_{L_{a}}+\tilde{u}_{L_{a}}^{*} \\
\tilde{u}_{R_{a}}+\tilde{u}_{R_{a}}^{*}
\end{array}\right\} \rightarrow \text { b jet }+ \text { jet }+\chi_{1}^{+}+\overline{\chi_{1}^{+}}, \\
p+p \rightarrow & \left\{\begin{array}{l}
\tilde{d}_{L_{a}}+\tilde{d}_{L_{a}}^{*} \\
\tilde{d}_{R_{a}}+\tilde{d}_{R_{a}}^{*}
\end{array}\right\} \rightarrow \text { top }+ \text { jet }+\chi_{1}^{+}+\overline{\chi_{1}^{+}} \\
& \rightarrow W+\text { b jet }+ \text { jet }+\chi_{1}^{+}+\overline{\chi_{1}^{+}} .
\end{aligned}
$$

The event then consists of two isolated charged tracks, two jets with one of them tagged as a 
$b$-jet, and no missing $\mathbb{E}_{T}$. As shown in Eqs. (12) and (13) additional leptons would be present from $W$ decay when the pair-produced squarks are of down-type flavor. The effective mass of all final state particles can be reconstructed to estimate the squark masses.

\section{Conclusions}

We have studied the production of the single top events at the LHC as a signal of flavor violation in the squark mass matrices of the MRSSM. The results may be summarized as follows:

- We considered the shortest decay chain where pair-produced squarks decay to quarks of different flavors and gauginos. The gaugino content of the lightest neutralino or chargino is either bino-like or Higgsino-like, given the constraint on $M_{2}$ [4]. Since the squarkHiggsino-quark coupling dominantly involves the third generation quarks, pair-produced squarks decay to pairs of tops whenever the lightest neutralino is mostly a Higgsino. As a result, the flavor-violating single top events are highly suppressed with respect to the double top events. Hence, the best case scenario to produce single top events is when the bino is the lightest neutralino.

- We performed a thorough analysis of the single top signal and background, when both neutralinos escape the detector. This is the so-called "collider equivalent LSP" Case I. We devise a set of cuts that reduce the background, achieving large "significance" of the single top signal. Furthermore, we estimated the significance of the signal as the squark mass and neutralino mass were varied.

- The best case scenario, our Case II, to detect flavor violation is when the neutralino decays within the detector to the gravitino and a photon. The signal contains two hard photons (generated at the interaction point or from secondary vertices, depending on the decay length of the neutralino) and provides virtually background free events. We explicitly showed how the size of the signal varies as the squark and neutralino masses were varied.

- In $R$-symmetric models, a chargino is often lighter than the lightest neutralino [44]. If the chargino is long-lived, it produces a charged track of a heavy object that will provide a nice handle to significantly reduce background events. While the production rate of flavor-violating signals suffers (the squark-Higgsino-quark coupling is dominated by the third generation)the unusual character of a long-lived chargino may well provide a means to identify a flavor-violating signal.

\section{Acknowledgments}

We thank Tim Tait and Carlos Wagner for very useful comments. GDK and AM thank the Aspen Center for Physics for hospitality where part of this work was completed. This work 
was supported in part by the DOE under contracts DE-FG02-96ER40969 (GDK, TSR) and DE-FG02-92ER40704 (AM).

\section{A Appendix: An R-symmetric Model, Mass Matrices, and the Interaction Lagrangian}

For the purpose of model building and calculations of various observables, the minimal $R$ symmetric supersymmetric model (MRSSM) is usually written in terms of two component Weyl fermions - in terms of which physics is simple and transparent. We however are interested in the collider phenomenology. Using modern Monte Carlo tools necessitates the construction of a formalism where all the fermions are in four component notation and all particles are represented by mass eigenstate fields. In this section we provide the notation and the details of one such formalism suitable for implementation into MadGraph. This section to be self-sufficient. Also, we only provide details of all the interactions that are of relevance for this paper (namely, fermion+sfermion+gaugino interactions).

\section{A.1 Particle Content}

First we list all the particles along with their quantum numbers under the standard model gauge group:

\begin{tabular}{c|cccc} 
Fields & $S U(3)_{C}$ & $S U(2)_{W}$ & $U(1)_{Y}$ & $U(1)_{R}$ \\
\hline$Q$ & 3 & 2 & $\frac{1}{6}$ & 1 \\
$U$ & $\overline{3}$ & 1 & $-\frac{2}{3}$ & 1 \\
$D$ & $\overline{3}$ & 1 & $\frac{1}{3}$ & 1 \\
$L$ & 1 & 2 & $-\frac{1}{2}$ & 1 \\
$E$ & 1 & 1 & 1 & 1 \\
$\Phi_{B}$ & 1 & 1 & 0 & 0 \\
$\Phi_{W}$ & 1 & 3 & 0 & 0 \\
$\Phi_{g}$ & 8 & 1 & 0 & 0 \\
$H_{u}$ & 1 & 2 & $\frac{1}{2}$ & 0 \\
$H_{d}$ & 1 & 2 & $-\frac{1}{2}$ & 0 \\
$R_{u}$ & 1 & 2 & $-\frac{1}{2}$ & 2 \\
$R_{d}$ & 1 & 2 & $\frac{1}{2}$ & 2 \\
\hline
\end{tabular}

Table A.1: Gauge and $R$-charges of all chiral supermultiplets in the MRSSM.

Table A.1 only contains the matter and Higgs superfields. In addition, the MRSSM also contains three real superfields $B, W$ and $G$ which are in the adjoint representations of corresponding gauge groups $\left(U(1)_{Y}, S U(2)_{W}, S U(3)_{C}\right.$ respectively) and carry zero $R$-charge. 
Next we look in the details of these multiplets. We establish notation for the components of the superfields, and write the mass matrices and interactions of interest.

\section{A.1.1 Gauge and Higgs Superfields}

The scalars and fermions in the Higgs superfields are denoted as

$$
\begin{array}{rlr}
H_{u}=\left(\begin{array}{c}
\left\{\tilde{H}_{u}^{+}, h_{u}^{+}\right\} \\
\left\{\tilde{H}_{u}^{0}, h_{u}^{0}\right\}
\end{array}\right), & H_{d}=\left(\begin{array}{c}
\left\{\tilde{H}_{d}^{0}, h_{d}^{0}\right\} \\
\left\{\tilde{H}_{d}^{-}, h_{d}^{-}\right\}
\end{array}\right), \\
R_{u}=\left(\begin{array}{c}
\left\{\tilde{R}_{u}^{0}, r_{u}^{0}\right\} \\
\left\{\tilde{R}_{u}^{-}, r_{u}^{-}\right\}
\end{array}\right), & R_{d}=\left(\begin{array}{c}
\left\{\tilde{R}_{d}^{+}, r_{d}^{+}\right\} \\
\left\{\tilde{R}_{d}^{0}, r_{d}^{0}\right\}
\end{array}\right) .
\end{array}
$$

The $R$-Higgs superfields are introduced to generate Higgsino masses in a $R$-symmetric way. The real superfields for the three gauge groups contain the usual spin- 1 gauge fields and their corresponding gauginos:

$$
\begin{array}{rlrl}
B & \rightarrow\left\{B_{\mu}, \tilde{B}\right\}, & W^{a} \rightarrow\left\{W_{\mu}^{a}, \tilde{W}^{a}\right\}, \quad G^{a} \rightarrow\left\{G_{\mu}^{a}, \tilde{G}^{a}\right\}, \\
\Phi_{B} & \rightarrow\left\{\psi_{B}, \phi_{B}\right\}, \quad \Phi_{W}^{a} \rightarrow\left\{\psi_{W}^{a}, \phi_{W}^{a}\right\}, \quad \Phi_{G}^{a} \rightarrow\left\{\psi_{G}^{a}, \phi_{G}^{a}\right\}
\end{array}
$$

All fermions shown in Eqs. A-1 and A-3 are Weyl fermions in the $\left(\frac{1}{2}, 0\right)$ representation of the Lorentz group. Combining the two-component spinors into four-component Dirac spinors, we have

$$
\begin{array}{r}
\lambda_{G}^{a}=\left(\begin{array}{c}
\psi_{G}^{a} \\
\tilde{G}^{a^{\dagger}}
\end{array}\right) \\
\lambda_{B}=\left(\begin{array}{c}
\psi_{B} \\
\tilde{B}^{\dagger}
\end{array}\right), \quad \lambda_{3}=\left(\begin{array}{c}
\psi_{W}^{0} \\
\tilde{W}^{0^{\dagger}}
\end{array}\right), \quad \lambda_{H_{1}}=\left(\begin{array}{c}
\tilde{H}_{u}^{0} \\
\tilde{R}_{u}^{0^{\dagger}}
\end{array}\right), \quad \lambda_{H_{2}}=\left(\begin{array}{c}
\tilde{H}_{d}^{0} \\
\tilde{R}_{d}^{0^{\dagger}}
\end{array}\right) \\
\lambda_{1}^{+}=\left(\begin{array}{c}
\psi_{W}^{+} \\
\tilde{W}^{-\dagger}
\end{array}\right), \quad \lambda_{2}^{-}=\left(\begin{array}{c}
\psi_{W}^{-} \\
\tilde{W}^{+}
\end{array}\right), \quad \lambda_{H_{1}}^{+}=\left(\begin{array}{c}
\tilde{H}_{u}^{+} \\
\tilde{R}_{u}^{-\dagger}
\end{array}\right), \quad \lambda_{H_{2}}^{-}=\left(\begin{array}{c}
\tilde{H}_{d}^{-} \\
\tilde{R}_{d}^{+}
\end{array}\right) .
\end{array}
$$

Note we have defined the four component spinors to be eigenstates of $R$-symmetry. In particular, all the four component fermions shown in Eqs. A-5, (A-6) and (A-7), which are also gauge eigenstates, have $R$-charge -1 . The charge conjugates of all the Dirac spinors above are defined in the usual way,

$$
\lambda^{c}=i \gamma_{2} \lambda^{*} \quad \text { and } \quad \lambda^{-}=i \gamma_{2}\left(\lambda^{+}\right)^{*}
$$

Next we list all operators which generate gaugino and the Higgsino mass terms:

$$
\begin{aligned}
\int d^{2} \theta \frac{W_{\alpha}^{\prime}}{M} W_{i}^{\alpha} \Phi_{i}+\mu_{u} H_{u} R_{u}+\mu_{d} & H_{d} R_{d} \\
& \quad+H_{u}\left(\lambda_{u} \Phi_{W}+\lambda_{u}^{\prime} \Phi_{B}\right) R_{u}+H_{d}\left(\lambda_{d} \Phi_{W}+\lambda_{d}^{\prime} \Phi_{B}\right) R_{d} .
\end{aligned}
$$

where $W_{\alpha}^{\prime}$ is the field strength chiral superfield for an extra $\mathrm{U}(1)$ and $i$ runs over the three standard model gauge groups $B, W$ and $G$. Gauginos and Higgsinos obtain their masses after 
$W_{\alpha}^{\prime}$ is expanded around its supersymmetry breaking VEV and $H_{u}$ and $H_{d}$ are expanded around their electroweak symmetry breaking VEV. Note that these are the only operators, involving the $R$ fields, that are allowed under all symmetries.

In our gauge eigenstate basis the neutralino masses may now be written as

$$
=\left[\begin{array}{llll}
\bar{\lambda}_{B} & \bar{\lambda}_{3} & \bar{\lambda}_{H_{1}} & \bar{\lambda}_{H_{2}}
\end{array}\right]\left[\begin{array}{cccc}
M_{1} & \bar{N} M_{N} P_{L} N+\text { c.c. } \\
0 & 0 & g^{\prime} v_{u} / \sqrt{2} & -g^{\prime} v_{d} / \sqrt{2} \\
\lambda_{u}^{\prime} v_{u} / \sqrt{2} & -\lambda_{u} v_{u} / \sqrt{2} & -g v_{u} / \sqrt{2} & g v_{d} / \sqrt{2} \\
-\lambda_{d}^{\prime} v_{d} / \sqrt{2} & \lambda_{d} v_{d} / \sqrt{2} & \mu_{u} & 0 \\
P_{L} & 0 & \mu_{d}
\end{array}\right] P_{L}\left[\begin{array}{c}
\lambda_{B} \\
\lambda_{3} \\
\lambda_{H_{1}} \\
\lambda_{H_{2}}
\end{array}\right]+\text { c.c. , }
$$

where $P_{L}$ and $P_{R}$ are the projection matrices on the 4 component Dirac spinors and projects out the top and the bottom Weyl spinors respectively. Although the vector $N$ of fermions and the mass matrix in Eq. A-10 looks similar to the neutralino mass term in the MSSM, they are drastically different. The vector $N$ is made of 4 component spinors, which are eigenstates of $R$-symmetry with $R$-charge -1 . The fact that the neutralinos carry a conserved $U(1)$ charge $(R$-symmetry in this case) makes them pure Dirac spinors.

Because of the Dirac nature of the neutralinos, their masses are determined by a biunitary diagonalization of the mass matrix $M_{N}$. This is entirely analogous to the chargino mass matrix. The squares of the neutralino masses are determined from diagonalizing the matrix $M_{N}^{\dagger} M_{N}$. In short, in the mass basis,

$$
\begin{gathered}
\bar{\chi} M_{\chi} P_{L} \chi+\text { c.c. } \\
\chi=\left(L_{N} P_{L}+R_{N} P_{R}\right) N \text { and } M_{\chi}=L_{N} M_{N} R_{N}^{\dagger},
\end{gathered}
$$

where $\chi$ are mass eigenstates and $M_{\chi}$ is a diagonal matrix with the diagonal entrees sorted. The unitary rotations $L_{N}$ and $R_{N}$ diagonalize and then sort $M_{N} M_{N}^{\dagger}$ and $M_{N}^{\dagger} M_{N}$ respectively. $P_{L}$ and $P_{R}$ are the projection matrices on the 4 component Dirac spinors and projects out the top and the bottom Weyl spinors respectively.

Similarly, the charginos of the MRSSM are written in terms of the gauge eigenstates as:

$$
\begin{gathered}
\bar{C} M_{C} P_{L} C^{+}+\text {c.c. } \\
=\left[\begin{array}{llll}
\bar{\lambda}_{1}^{+} & \bar{\lambda}_{H_{1}}^{+} & \bar{\lambda}_{2}^{+} & \bar{\lambda}_{H_{2}}^{+}
\end{array}\right]\left[\begin{array}{cccc}
M_{2} & g v_{u} & 0 & 0 \\
\lambda_{u} v_{u} & \mu_{u} & 0 & 0 \\
0 & 0 & M_{2} & \lambda_{d} v_{d} \\
0 & 0 & g v_{d} & \mu_{d}
\end{array}\right] P_{L}\left[\begin{array}{c}
\lambda_{1}^{+} \\
\lambda_{H_{1}}^{+} \\
\lambda_{2}^{+} \\
\lambda_{H_{2}}^{+}
\end{array}\right]+\text {c.c. } .
\end{gathered}
$$

Just like the neutralinos, Eq. A-13 can be written in terms of the mass eigenstates

$$
\begin{gathered}
\overline{\chi^{+}} M_{\chi^{+}} P_{L} \chi^{+}+\text {c.c. } \\
\chi^{+}=\left(L_{C} P_{L}+R_{C} P_{R}\right) C^{+} \text {and } M_{\chi^{+}}=L_{C} M_{C} R_{C}^{\dagger},
\end{gathered}
$$




\section{A.1.2 Matter Superfields}

We take the matter superfields to be:

$$
\begin{gathered}
Q \rightarrow\left(\begin{array}{c}
\left\{\xi_{u}, \tilde{u}_{L}\right\} \\
\left\{\xi_{d}, \tilde{d}_{L}\right\}
\end{array}\right), \quad U \rightarrow\left\{\xi_{\bar{u}}, \tilde{u}_{R}^{*}\right\}, \quad D \rightarrow\left\{\xi_{\bar{d}}, \tilde{d}_{R}^{*}\right\} \\
L \rightarrow\left(\begin{array}{c}
\left\{\xi_{\nu}, \tilde{\nu}_{L}\right\} \\
\left\{\xi_{e}, \tilde{e}_{L}\right\}
\end{array}\right), \quad E \rightarrow\left\{\xi_{\bar{e}}, \tilde{e}_{R}^{*}\right\}
\end{gathered}
$$

All $\xi_{f}$ are Weyl fermions in the $\left(\frac{1}{2}, 0\right)$ representation of the Lorentz group, while $\tilde{u}_{\lambda}, \tilde{d}_{\lambda}$, etc. are the superpartner scalars. We have defined the superfields $U, D$ and $E$ in such a way that both the left and the right scalar superpartners have the same charge.

For the purpose of phenomenology it is more convenient to use a notation where all the fermions are represented in a four component notation.

$$
u_{i}=\left(\begin{array}{c}
\xi_{u_{i}} \\
\bar{\xi}_{\bar{u}_{i}}
\end{array}\right), \quad d_{i}=\left(\begin{array}{c}
\xi_{d_{i}} \\
\bar{\xi}_{\bar{d}_{i}}
\end{array}\right), \quad l_{i}=\left(\begin{array}{c}
\xi_{\ell_{i}} \\
\bar{\xi}_{\bar{l}_{i}}
\end{array}\right), \quad \nu_{i}=\left(\begin{array}{c}
\xi_{\nu_{i}} \\
\bar{\xi}_{\bar{\nu}_{i}}
\end{array}\right)
$$

where $i$ is the generation index. Without loss of generality we will work in a basis where the Yukawa couplings are diagonal (the mass eigenbasis). For example, $\xi_{u_{i}}$ and $\xi_{\bar{u}_{i}}$ correspond to the $i$ th eigenstate of the up-type quark mass terms.

This is, however, not the mass basis of the scalar superpartners. Assuming that only the third generation fermions are massive, we find the mass terms of the matter superparticles are given by:

$$
m_{\tilde{f}_{i j}}^{2} \tilde{f}_{i}^{*} \tilde{f}_{j}=\left(\tilde{m}_{f_{i j}}^{2}+m_{f}^{2} \delta_{i 3} \delta_{j 3}\right) \tilde{f}_{i}^{*} \tilde{f}_{j}
$$

where $\tilde{f}$ runs over the species $\tilde{u}_{L}, \tilde{u}_{R}, \tilde{d}_{L}, \tilde{d}_{R}, \tilde{e}_{L}, \tilde{e}_{R}$ and $\tilde{\nu}_{L}$. In particular, mass parameters given in Eq. (A-18) are

$$
\begin{array}{r}
\tilde{m}_{u_{L}}^{2}=\tilde{m}_{d_{L}}^{2}=\tilde{m}_{Q}^{2}, \quad \tilde{m}_{u_{R}}^{2}=\tilde{m}_{U}^{2}, \quad \tilde{m}_{d_{R}}^{2}=\tilde{m}_{D}^{2}, \quad \tilde{m}_{e_{L}}^{2}=\tilde{m}_{\nu_{L}}^{2}=\tilde{m}_{L}^{2}, \quad \tilde{m}_{e_{R}}^{2}=\tilde{m}_{E}^{2} \\
m_{u_{L}}=m_{u_{R}}=m_{t}, \quad m_{d_{L}}=m_{d_{R}}=m_{b}, \quad m_{e_{L}}=m_{e_{R}}=m_{\tau}, \quad m_{\nu_{L}}=0,
\end{array}
$$

where we have taken the first and second generation SM quark/lepton masses to vanish. The physical masses $m_{\tilde{f}}$ are now diagonalized by unitary transformations. Each species $\tilde{f} \supset \tilde{u}_{L}, \tilde{d}_{L}, \tilde{u}_{R}, \tilde{d}_{R}, \tilde{e}_{L}, \tilde{e}_{R}$, and $\tilde{\nu}_{L}$ has its own transformation, which we designate as $U_{f}$. For convenience we designate scalars (sfermions) in mass basis by $\tilde{f}_{a}$, as opposed to $\tilde{f}_{i}$, which we used to designate sfermions in the basis where the Yukawa matrices (fermion mass matrices) are diagonal.

$$
\tilde{f}_{a}=U_{f_{a i}}^{\dagger} \tilde{f}_{i}
$$

\section{A.2 Gaugino-sfermion-fermion Interactions}

There are two sources of fermion-sfermion-gaugino interactions. First, these interactions arise in the superfield kinetic terms as a consequence of gauge-invariance. Therefore these interactions have gauge-coupling strength and couple gauginos to the fermions and the sfermions. The 
sfermions and the fermions in these interactions have the same chirality. The Yukawa terms in the superpotential are the second source of fermion-sfermion-gaugino interactions. The superpotential interactions couple Higgsinos to fermions and sfermions of opposite handedness.

\section{A.2.1 Gluino-fermion-sfermion Interactions}

In the basis where all the fermions and as well as the sfermions are mass eigenstates the interactions are given as:

$$
\begin{aligned}
-\sqrt{2} g_{s}\left[\left(\bar{\lambda}_{G} \tilde{u}_{L_{a}}^{*}\left(U_{u_{L}}\right)_{a i} P_{L}-\bar{\lambda}_{G}^{c} \tilde{u}_{R_{a}}^{*}\left(U_{u_{R}}\right)_{a i} P_{R}\right) u_{i}\right. \\
\left.+\left(\bar{\lambda}_{G} \tilde{d}_{L_{a}}^{*}\left(U_{d_{L}}\right)_{a i} P_{L}-\bar{\lambda}_{G}^{c} \tilde{d}_{R_{a}}^{*}\left(U_{d_{R}}\right)_{a i} P_{R}\right) d_{i}\right]+ \text { c.c. }
\end{aligned}
$$

where we have abbreviated $\lambda_{G} \equiv \lambda_{G}^{a} t_{G}^{a}$ and the matrices $t_{G}^{a}$ are the generators for the color group.

\section{A.2.2 Neutralino-sfermion-fermion Interactions}

This set of interactions are slightly more complicated because we need to take into account not only the rotations that take the scalars to their mass eigenstates, but also to change basis for the neutralinos. Resisting the temptation to write the final expression, we first write the interactions with neutralinos in the electroweak basis and sfermions in the fermion mass basis (i.e. in terms of $f_{i}$ ). Specifically, our expression below is in terms of the vector $N$, which can be thought of a column of Dirac fermions. We will also use four projection vectors $P_{1}, \ldots, P_{4}$ that project out corresponding components. For example, $\bar{\lambda}_{B}=\bar{N} P_{1}-$ here $\bar{N}$ is a row as shown in Eq. A-10) and $P_{1}$ is a column with zero elements everywhere except at the first row, which is 
1. The neutralino-fermion-sfermion interactions are now

$$
\begin{array}{r}
-\sqrt{2} g \tan \theta_{W}\left[\bar{N} P_{1}\left(\frac{1}{6} \tilde{u}_{L_{i}}^{*} \delta_{i j} P_{L} u_{j}+\frac{1}{6} \tilde{d}_{L_{i}}^{*} \delta_{i j} P_{L} d_{j}-\frac{1}{2} \tilde{e}_{L_{i}}^{*} \delta_{i j} P_{L} e_{j}-\frac{1}{2} \tilde{\nu}_{L_{i}}^{*} \delta_{i j} P_{L} \nu_{j}\right)\right. \\
\left.+\overline{N^{c}} P_{1}\left(-\frac{2}{3} \tilde{u}_{R_{i}}^{*} \delta_{i j} P_{R} u_{j}+\frac{1}{3} \tilde{d}_{R_{i}}^{*} \delta_{i j} P_{R} d_{j}+\tilde{e}_{R_{i}}^{*} \delta_{i j} P_{R} e_{j}\right)\right] \\
-\sqrt{2} g\left[\bar{N} P_{2}\left(\frac{1}{2} \tilde{u}_{L_{i}}^{*} \delta_{i j} P_{L} u_{j}-\frac{1}{2} \tilde{d}_{L_{i}}^{*} \delta_{i j} P_{L} d_{j}-\frac{1}{2} \tilde{e}_{L_{i}}^{*} \delta_{i j} P_{L} e_{j}+\frac{1}{2} \tilde{\nu}_{L_{i}}^{*} \delta_{i j} P_{L} \nu_{j}\right)\right] \\
-\sqrt{2} \frac{m_{t}}{v \sin \beta}\left[\left(\bar{N} P_{3} \tilde{u}_{L_{i}}^{*} \delta_{i 3} P_{R}+\overline{N^{c}} P_{3} \tilde{u}_{R_{i}}^{*} \delta_{i 3} P_{L}\right) u_{3}\right] \\
-\sqrt{2} \frac{m_{b}}{v \cos \beta}\left[\left(\bar{N} P_{4} \tilde{d}_{L_{i}}^{*} \delta_{i 3} P_{R}+\overline{N^{c}} P_{4} \tilde{d}_{R_{i}}^{*} \delta_{i 3} P_{L}\right) d_{3}\right] \\
-\sqrt{2} \frac{m_{\tau}}{v \cos \beta}\left[\left(\bar{N} P_{4} \tilde{e}_{L_{i}}^{*} \delta_{i 3} P_{R}+\overline{N^{c}} P_{4} \tilde{e}_{R_{i}}^{*} \delta_{i 3} P_{L}\right) e_{3}\right]+\text { c.c. }
\end{array}
$$

The advantage of using matrix notation is now evident. Neutralinos and scalar sfermions in their mass basis are related to the ones in Eq. A-21) by $\bar{N}=\bar{\chi}\left(L_{N} P_{L}+R_{N} P_{R}\right)$ and $\tilde{f}_{i}^{*}=\tilde{f}_{a}^{*} U_{f_{a i}}$. The final expressions are:

$$
\begin{aligned}
& -\sqrt{2} g \tan \theta_{W}\left[\overline { \chi } L _ { N } P _ { 1 } \left(\frac{1}{6} \tilde{u}_{L_{a}}^{*}\left(U_{\tilde{u}_{L}}\right)_{a j} P_{L} u_{j}+\frac{1}{6} \tilde{d}_{L_{a}}^{*}\left(U_{\tilde{d}_{L}}\right)_{a j} P_{L} d_{j}\right.\right. \\
& \left.-\frac{1}{2} \tilde{e}_{L_{a}}^{*}\left(U_{\tilde{e}_{L}}\right)_{a j} P_{L} e_{j}-\frac{1}{2} \tilde{\nu}_{L_{a}}^{*}\left(U_{\tilde{\nu}_{L}}\right)_{a j} P_{L} \nu_{j}\right) \\
& +\overline{\chi^{c}} L_{N} P_{1}\left(-\frac{2}{3} \tilde{u}_{R_{a}}^{*}\left(U_{\tilde{u}_{R}}\right)_{a j} P_{R} u_{j}+\frac{1}{3} \tilde{d}_{R_{a}}^{*}\left(U_{\tilde{d}_{R}}\right)_{a j} P_{R} d_{j}\right. \\
& \left.\left.+\tilde{e}_{R_{a}}^{*}\left(U_{\tilde{e}_{R}}\right)_{a j} P_{R} e_{j}\right)\right] \\
& -\sqrt{2} g\left[\overline { \chi } L _ { N } P _ { 2 } \left(\frac{1}{2} \tilde{u}_{L_{a}}^{*}\left(U_{\tilde{u}_{L}}\right)_{a j} P_{L} u_{j}-\frac{1}{2} \tilde{d}_{L_{a}}^{*}\left(U_{\tilde{d}_{L}}\right)_{a j} P_{L} d_{j}\right.\right. \\
& \left.\left.-\frac{1}{2} \tilde{e}_{L_{a}}^{*}\left(U_{\tilde{e}_{L}}\right)_{a j} P_{L} e_{j}+\frac{1}{2} \tilde{\nu}_{L_{a}}^{*}\left(U_{\tilde{\nu}_{L}}\right)_{a j} P_{L} \nu_{j}\right)\right] \\
& -\sqrt{2} \frac{m_{t}}{v \sin \beta}\left[\left(\bar{\chi} R_{N} P_{3} \tilde{u}_{L_{a}}^{*}\left(U_{\tilde{u}_{L}}\right)_{a 3} P_{R}+\overline{\chi^{c}} R_{N} P_{3} \tilde{u}_{R_{a}}^{*}\left(U_{\tilde{u}_{R}}\right)_{a 3} P_{L}\right) u_{3}\right] \\
& -\sqrt{2} \frac{m_{b}}{v \cos \beta}\left[\left(\bar{\chi} R_{N} P_{4} \tilde{d}_{L_{a}}^{*}\left(U_{\tilde{d}_{L}}\right)_{a 3} P_{R}+\overline{\chi^{c}} R_{N} P_{4} \tilde{d}_{R_{a}}^{*}\left(U_{\tilde{d}_{R}}\right)_{a 3} P_{L}\right) d_{3}\right] \\
& -\sqrt{2} \frac{m_{\tau}}{v \cos \beta}\left[\left(\bar{\chi} R_{N} P_{4} \tilde{e}_{L_{a}}^{*}\left(U_{\tilde{e}_{L}}\right)_{a 3} P_{R}+\overline{\chi^{c}} R_{N} P_{4} \tilde{e}_{R_{a}}^{*}\left(U_{\tilde{e}_{R}}\right)_{a 3} P_{L}\right) e_{3}\right]+\text { c.c. . }
\end{aligned}
$$




\section{A.2.3 Chargino-sfermion-fermion Interactions}

Deriving the chargino-fermion-sfermion expressions is entirely analogous to the previous exercise where we found the interactions with the neutralinos. Here we just give the final results where all the fields involved are mass eigenstates. The chargino-quark-squark interactions already violate flavor, even if the quark and squark mass matrices are diagonalized simultaneously, due to the CKM matrix $V$. Additional rotations of the squarks changes the amount of flavor violation.

$$
\begin{aligned}
-g\left[\overline{\chi^{-}} L_{C} P_{3}\left(\tilde{u}_{L_{a}}^{*}\left(U_{\tilde{u}_{L}} V^{\dagger}\right)_{a j} P_{L} d_{j}+\tilde{\nu}_{L_{a}}^{*}\left(U_{\tilde{\nu}_{L}}\right)_{a j} P_{L} e_{j}\right)\right. \\
\left.+\overline{\chi^{+}} R_{C} P_{1}\left(\tilde{d}_{L_{a}}^{*}\left(U_{\tilde{d}_{L}} V\right)_{a j} P_{L} u_{j}+\tilde{e}_{L_{a}}^{*}\left(U_{\tilde{e}_{L}}\right)_{a j} P_{L} \nu_{j}\right)\right] \\
+\sqrt{2} \frac{m_{t}}{v \sin \beta}\left[\overline{\chi^{+}} L_{C} P_{2} \tilde{d}_{L_{a}}^{*}\left(U_{\tilde{d}_{L}} V\right)_{a 3} P_{R} u_{3}+\overline{\chi^{-}} L_{C} P_{3} \tilde{u}_{R_{a}}^{*}\left(U_{\tilde{u}_{R}} V^{\dagger}\right)_{a 3} P_{L} d_{3}\right] \\
+\sqrt{2} \frac{m_{b}}{v \cos \beta}\left[\overline{\chi^{-}} R_{C} P_{4} \tilde{u}_{L_{a}}^{*}\left(U_{\tilde{u}_{L}} V^{\dagger}\right)_{a 3} P_{R} d_{3}+\overline{\chi^{+}} R_{C} P_{4} \tilde{d}_{R_{a}}^{*}\left(U_{\tilde{d}_{R}} V\right)_{a 3} P_{L} u_{3}\right] \\
+\sqrt{2} \frac{m_{\tau}}{v \cos \beta}\left[\overline{\chi^{-}} R_{C} P_{4} \tilde{\nu}_{L_{a}}^{*}\left(U_{\tilde{\nu}_{L}}\right)_{a 3} P_{R} e_{3}+\overline{\chi^{+}} R_{C} P_{4} \tilde{e}_{R_{a}}^{*}\left(U_{\tilde{e}_{R}}\right)_{a 3} P_{L} \nu_{3}\right]+\text { c.c. . }
\end{aligned}
$$

\section{References}

[1] J. R. Ellis and D. V. Nanopoulos, Phys. Lett. B 110, 44 (1982).

[2] J. F. Donoghue, H. P. Nilles and D. Wyler, Phys. Lett. B 128, 55 (1983).

[3] For an older review, see F. Gabbiani, E. Gabrielli, A. Masiero and L. Silvestrini, Nucl. Phys. B 477, 321 (1996) arXiv:hep-ph/9604387.

[4] G. D. Kribs, E. Poppitz and N. Weiner, Phys. Rev. D 78, 055010 (2008) arXiv:0712.2039 [hep-ph]].

[5] A. E. Blechman and S. P. Ng, JHEP 0806, 043 (2008) arXiv:0803.3811 [hep-ph]].

[6] T. Plehn and T. M. P. Tait, arXiv:0810.3919 [hep-ph].

[7] For a review, see e.g. W. Bernreuther, J. Phys. G 35, 083001 (2008) arXiv:0805.1333 [hep-ph]].

[8] T. M. P. Tait and C. P. P. Yuan, Phys. Rev. D 63, 014018 (2001) arXiv:hep-ph/0007298.

[9] J. J. Liu, C. S. Li, L. L. Yang and L. G. Jin, Nucl. Phys. B 705, 3 (2005) arXiv:hep$\mathrm{ph} / 0404099$. 
[10] J. Guasch, W. Hollik, S. Penaranda and J. Sola, Nucl. Phys. Proc. Suppl. 157, 152 (2006) arXiv:hep-ph/0601218.

[11] G. Eilam, M. Frank and I. Turan, Phys. Rev. D 74, 035012 (2006) arXiv:hep-ph/0601253.

[12] J. J. Cao, G. Eilam, M. Frank, K. Hikasa, G. L. Liu, I. Turan and J. M. Yang, Phys. Rev. D 75, 075021 (2007) |arXiv:hep-ph/0702264.

[13] G. Bozzi, B. Fuks, B. Herrmann and M. Klasen, Nucl. Phys. B 787, 1 (2007) arXiv:0704.1826 [hep-ph]].

[14] D. Lopez-Val, J. Guasch and J. Sola, JHEP 0712, 054 (2007) arXiv:0710.0587 [hep-ph]].

[15] S. Bejar, J. Guasch, D. Lopez-Val and J. Sola, Phys. Lett. B 668, 364 (2008) arXiv:0805.0973 [hep-ph]].

[16] A. Datta, J. M. Yang, B. L. Young and X. Zhang, Phys. Rev. D 56, 3107 (1997) arXiv:hepph/9704257.

[17] R. J. Oakes, K. Whisnant, J. M. Yang, B. L. Young and X. Zhang, Phys. Rev. D 57, 534 (1998) arXiv:hep-ph/9707477.

[18] E. L. Berger, B. W. Harris and Z. Sullivan, Phys. Rev. Lett. 83, 4472 (1999) arXiv:hep$\mathrm{ph} / 9903549$.

[19] E. L. Berger, B. W. Harris and Z. Sullivan, Phys. Rev. D 63, 115001 (2001) arXiv:hepph/0012184.

[20] For a review, see G. F. Giudice and R. Rattazzi, Phys. Rept. 322, 419 (1999) arXiv:hep$\mathrm{ph} / 9801271$.

[21] A. G. Cohen, T. S. Roy and M. Schmaltz, JHEP 0702, 027 (2007) arXiv:hep-ph/0612100.

[22] T. S. Roy and M. Schmaltz, Phys. Rev. D 77, 095008 (2008) arXiv:0708.3593 [hep-ph]].

[23] H. Murayama, Y. Nomura and D. Poland, Phys. Rev. D 77, 015005 (2008) arXiv:0709.0775 [hep-ph]].

[24] G. L. Bayatian et al. [CMS Collaboration], J. Phys. G 34, 995 (2007).

[25] R. Harnik and G. D. Kribs, arXiv:0810.5557 [hep-ph].

[26] O. Adriani et al., arXiv:0810.4995 [astro-ph].

[27] M. L. Mangano, M. Moretti, F. Piccinini, R. Pittau and A. D. Polosa, JHEP 0307, 001 (2003) arXiv:hep-ph/0206293.

[28] F. Maltoni and T. Stelzer, JHEP 0302, 027 (2003) arXiv:hep-ph/0208156.

[29] J. Alwall et al., JHEP 0709, 028 (2007) arXiv:0706.2334 [hep-ph]].

[30] T. Sjostrand, S. Mrenna and P. Skands, JHEP 0605, 026 (2006) arXiv:hep-ph/0603175. 
[31] J. Conway, http://www.physics.ucdavis.edu/ conway/research/software/pgs/pgs4general.htm

[32] G. H. Brooijmans et al., arXiv:0802.3715 [hep-ph].

[33] S. Catani, F. Krauss, R. Kuhn and B. R. Webber, JHEP 0111, 063 (2001) arXiv:hep$\mathrm{ph} / 0109231$.

[34] H. C. Cheng, D. Engelhardt, J. F. Gunion, Z. Han and B. McElrath, Phys. Rev. Lett. 100, 252001 (2008) arXiv:0802.4290 [hep-ph]].

[35] H. C. Cheng, J. F. Gunion, Z. Han, G. Marandella and B. McElrath, JHEP 0712, 076 (2007) arXiv:0707.0030 [hep-ph]].

[36] M. Burns, K. Kong, K. T. Matchev and M. Park, arXiv:0810.5576 [hep-ph].

[37] C. Athanasiou, C. G. Lester, J. M. Smillie and B. R. Webber, JHEP 0608, 055 (2006) arXiv:hep-ph/0605286.

[38] L. T. Wang and I. Yavin, JHEP 0704, 032 (2007) arXiv:hep-ph/0605296.

[39] M. T. Bowen, Phys. Rev. D 73, 097501 (2006) arXiv:hep-ph/0503110].

[40] ATLAS detector and physics performance. Technical design report. Vol. 2, (1999).

[41] D. Prieur, ATL-PHYS-PUB-2007-010. ATL-COM-PHYS-2007-013.

[42] G. Aad et al. [The ATLAS Collaboration], arXiv:0901.0512.

[43] S. Ambrosanio, G. L. Kane, G. D. Kribs, S. P. Martin and S. Mrenna, Phys. Rev. D 54, 5395 (1996) arXiv:hep-ph/9605398.

[44] G. D. Kribs, A. Martin and T. S. Roy, JHEP 0901, 023 (2009) arXiv:0807.4936 [hep-ph]].

[45] C. Amsler et al. [Particle Data Group], Phys. Lett. B 667, 1 (2008).

[46] A. Nisati, S. Petrarca and G. Salvini, Mod. Phys. Lett. A 12, 2213 (1997) arXiv:hep$\mathrm{ph} / 9707376$.

[47] B. C. Allanach, C. M. Harris, M. A. Parker, P. Richardson and B. R. Webber, JHEP 0108, 051 (2001) arXiv:hep-ph/0108097.

[48] J. Ellis, A. Raklev, and O. Oye, ATL-PHYS-PUB-2007-016. ATL-COM-PHYS-2006-093.

[49] S. Giagu [ATLAS Collaboration and CMS Collaboration], arXiv:0810.1453 [hep-ex]. 